Pacific Journal of Mathematics

A FUNCTIONAL CALCULUS FOR BANACH PI-ALGEBRAS 


\section{A FUNCTIONAL CALCULUS FOR BANACH PI-ALGEBRAS}

\section{Denis LuMinet}

Let $A$ be a unital Banach algebra. Take $a_{1}, \ldots, a_{n} \in A$ and let $B$ be the closed subalgebra of $A$ they generate. The algebras $\mathscr{D}(\Omega)$ of entire matrix-valued functions were introduced by $J$. L. Taylor, who asked if they led to a functional calculus, generalizing the ShilovWaelbroeck-Arens-Calderon theorem. We show that a necessary condition for a functional calculus map $\mathscr{D}(\Omega) \rightarrow A$ to exist is that $B$ satisfy a polynomial identity; sufficient conditions are that $B$ be a topological subquotient of a Banach Azumaya algebra, or that $n=2$ and $B$ satisfy all identities of $2 \times 2$ matrices. For closed subalgebras of Banach Azumaya algebras, we obtain a functional calculus on polynomial polyhedra containing the joint spectrum. Various properties of algebras of matrix-valued functions are studied, including domains of holomorphy.

Introduction. In the early 1950's, Shilov, Waelbroeck and ArensCalderon constructed a functional calculus for several variables in a commutative Banach algebra with unit (all algebras are assumed to be complex). If $A$ is such an algebra, and $a_{1}, \ldots, a_{n} \in A$, we have a homomorphism $P_{n} \rightarrow A$ that sends $1 \mapsto 1, X_{1} \mapsto a_{1}, \ldots, X_{n} \mapsto a_{n}$, where $P_{n}$ is the polynomial algebra in $X_{1}, \ldots, X_{n}$. This map extends to a continuous homomorphism $\mathcal{O}(U) \rightarrow A$, i.e., we have a commutative diagram

$$
\begin{gathered}
P_{n} \rightarrow A \\
\searrow \nearrow \\
\mathcal{O}(U)
\end{gathered}
$$

provided the open subset $U$ of $\mathbf{C}^{n}$ contains the joint spectrum of $\left(a_{1}, \ldots, a_{n}\right)[8]$.

Is there a reasonable equivalent of this result for noncommutative algebras? Let $A$ be any unital Banach algebra, and take $a_{1}, \ldots, a_{n} \in A$. We have a homomorphism $F_{n} \rightarrow A$ that sends $1 \mapsto 1, X_{1} \mapsto a_{1}, \ldots, X_{n} \mapsto$ $a_{n}$, where $F_{n}$ is the free algebra in $X_{1}, \ldots, X_{n}$. Can we extend this map to some algebra $\mathscr{A}$ of "noncommutative functions"

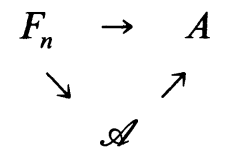


the existence of the extension being subordinate to a condition on a (yet to be defined) joint spectrum of $\left(a_{1}, \ldots, a_{n}\right)$ ? This is the functional calculus problem for noncommutative algebras.

Our first task is to define the algebras of noncommutative functions that will play the role of the $\mathcal{O}(U), U \subseteq \mathbf{C}^{n}$. Let us again consider a commutative situation: entire functions of one variable. We can regard them as "holomorphic functions", i.e., continuously differentiable functions $\mathbf{C} \rightarrow \mathbf{C}$ that satisfy the Cauchy-Riemann equations. Or we can view them as "analytic functions", i.e., power series with infinite radius of convergence. Of course, these notions are identical: a function is holomorphic if and only if it is analytic. The equivalence holds for functions of $n$ variables; any function holomorphic (in the Cauchy-Riemann sense) in the open set $U \subseteq \mathbf{C}^{n}$ is locally the sum of its Taylor series, and conversely.

It is somewhat surprising that the natural generalizations of "holomorphic" and "analytic" to noncommuting variables give rise to two different theories. For simplicity, we shall deal again with "entire" functions. We can consider functions that send $n$-tuples of $i \times i$ matrices to $i \times i$ matrices ( $n \geq 2$ is fixed, $i$ ranges over the positive integers). If we demand that these functions satisfy suitable intertwining conditions, we obtain the algebra $\mathscr{D}(\Omega)$ of "free entire holomorphic functions". Even simpler is the definition of $\mathscr{F}(\infty)$, the algebra of "free entire analytic functions": we take all free power series with infinite radius of convergence. J. L. Taylor, who introduced $\mathscr{D}(\Omega)$ and $\mathscr{F}(\infty)$ in [14], showed that $\mathscr{F}(\infty)$ embeds in $\mathscr{D}(\Omega)$. The inclusion is proper: take free indeterminates $X_{1}$ and $X_{2}$, and consider

$$
f\left(X_{1}, X_{2}\right)=\sum_{k=2}^{\infty} S_{k}\left(X_{1}, X_{1} X_{2}, \ldots, X_{1} X_{2}^{k-1}\right)
$$

where $S_{k}$ is the standard alternating free polynomial in $k$ variables. It is easily shown that $f \in \mathscr{D}(\Omega)$, for the value of $f$ on a couple of matrices is always given by a finite sum (Amitsur-Levitzki theorem, see [10]); but $f \notin \mathscr{F}(\infty)$ : it cannot be written as a convergent power series (2.9).

Let us go back to the functional calculus problem. Since we can always substitute Banach algebra elements in a free power series, the map $F_{n} \rightarrow A$ extends to $\mathscr{F}(\infty)$. However, we are looking for a functional calculus, and $\mathscr{F}(\infty)$ is not really a function algebra. Thus, we now ask whether the map $F_{n} \rightarrow A$ extends to $\mathscr{D}(\Omega)$ (which is a function algebra)

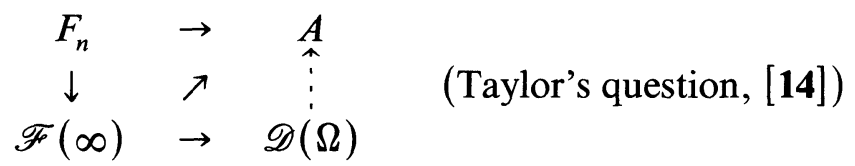


In order for the dotted map to exist, it is necessary that the subalgebra of $A$ generated by $a_{1}, \ldots, a_{n}$ be a PI-algebra, i.e., some free polynomial vanishes identically when evaluated on elements of the algebra (2.7).

The converse question is more delicate. We conjecture that $\mathscr{D}(\Omega)$ gives a functional calculus for all PI-algebras and any (finite) number of variables. We prove this

(a) when the algebra is a separated quotient of a closed subalgebra of a Banach Azumaya algebra;

(b) when the number of variables is two, and the algebra satisfies all polynomial identities of $2 \times 2$ matrices.

It is not known if every finitely generated Banach PI-algebra enjoys property (a). Even if this is not the case, (b) indicates that (a) might be superfluous.

To attack this question, we shall assume henceforth that the unital Banach algebra $A$ satisfies all identities of $i \times i$ matrices. Then the map $F_{n} \rightarrow A$ vanishes on the ideal $T_{n, i}$ of those elements in $F_{n}$ that are polynomial identities for $i \times i$ matrices, and yields a map $F_{n} / T_{n, i}=R_{n, i}$ $\rightarrow A$, where $R_{n, i}$ is the algebra of $n$ generic $i \times i$ matrices. $R_{n, i}$ can also be described as an algebra of polynomial functions $M_{i}^{n} \rightarrow M_{i}\left(M_{i}\right.$ is the algebra of complex $i \times i$ matrices; likewise $M_{i j}$ is the space of complex $i \times j$ matrices, $\mathrm{GL}_{i}$ the complex general linear group,...). We can consider $\mathscr{R}$, the closure of $R_{n, i}$ in $\mathcal{O}\left(M_{i}^{n}, M_{i}\right)$. An intrinsic definition of $\mathscr{R}$ can be given for $n=i=2$, but fails when $i>2$ or $n>2$. $\mathscr{R}$ is the algebra of "entire holomorphic functions of $n i \times i$ matrices". Does $R_{n, i} \rightarrow A$ extend to $\mathscr{R}$ ?

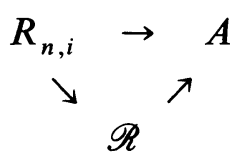

This question is equivalent to the following: can every function in $\mathscr{R}$ be represented by a power series, i.e., do "holomorphic" and "analytic" coincide for entire functions of $n i \times i$ matrices (where now both $n$ and $i$ are fixed). Still another way to phrase the question is: do $\mathscr{D}(\Omega)$ and $\mathscr{F}(\infty)$ agree after factoring out the functions that vanish on $i \times i$ matrices? If the answer is affirmative, we will have shown that $\mathscr{D}(\Omega) \rightarrow A$.

When $n=i=2$, we can prove it, because we have precise structure results on $R_{2,2}$, the algebra of two generic $2 \times 2$ matrices [7]. Our approach does not seem to generalize to $n>2$ or $i>2$, when the structure of the generic matrix algebra is less well understood. The basic difficulty with our algebras $\mathscr{R}$ is that they are not finite modules over 
their centers, and the centers are not finitely generated algebras. Nevertheless, we can try a more complicated construction. Instead of considering entire free power series, we take free power series with a finite polyradius of convergence. Also, we add $q$ extra indeterminates to the $n$ we started with, and impose $q$ relations, before factoring out the identities of $i \times i$ matrices. If the relations are chosen suitably, the quotient turns out to be an Azumaya algebra (hence finite over its center), and its center is finitely generated. Then we can show that the quotient is isomorphic to the algebra of all "holomorphic functions" defined on an open set of $M_{l}^{n}$ which is a natural generalization of a polynomial polyhedron. This implies the existence of $\mathscr{D}(\Omega) \rightarrow A$ when $A$ is (a topological subquotient of) an Azumaya algebra ( $n, i$ arbitrary).

The contents of this paper can be summarized as follows. In Chapter One, we give basic facts about algebras with polynomial identities. The sole original result is a description of the algebra of two generic $2 \times 2$ matrices by intertwining conditions. Free algebras of analytic and holomorphic functions are introduced in Chapter Two, which is based on Taylor's [14]. We include a study of "domains of holomorphy", parallel to the domains of holomorphy in classical complex analysis. In Chapter Three, we discuss PI-algebras of holomorphic functions. Chapters Four and Five, where the commutative functional calculus plays an important role, constitute the heart of the paper. In Chapter Four, after developing an implicit function theorem due to Taylor, we show that holomorphic functions on $n i \times i$ matrices admits power series expressions in appropriate domains, for which the function algebras are Azumaya. Chapter Five concentrates on functions of two $2 \times 2$ matrices. Finally, Chapter Six describes the construction of a functional calculus, under the assumptions (a) or (b) stated on page 129. For closed subalgebras of Azumaya algebras, we obtain a functional calculus on polynomial polyhedra containing the spectrum.

This paper originates in a doctoral dissertation completed at the University of Utah under the supervision of Joseph L. Taylor, whom we thank for his encouragement and stimulating comments.

\section{Chapter One. Polynomial Identities}

Let $A$ be a complex algebra with unit. $A$ satisfies a polynomial identity if some noncommutative polynomial in $n$ variables vanishes on all $n$-tuples of elements of $A$. We also say that $A$ is a PI-algebra. We shall 
write $A \leq M_{i}$ when $A$ satisfies all the identities of $i \times i$ matrices (in particular the standard identity $S_{2 i}=0$ ).

Basic properties of PI-algebras can be found in [9], [12], [16]. Let us also mention this recent result.

1.1. Proposition (Braun). If $A$ is a finitely generated PI-algebra, there exists $i \in \mathbf{N}$ such that $A \leq M_{i}$.

Proof. See [4].

Azumaya algebras (also called central separable algebras) form an important class of PI-algebras. Here is a deep and useful fact.

1.2. Proposition (Artin-Procesi). A is an Azumaya algebra of rank $i^{2}$ over its center $Z$ if and only if

(a) $A \leq M_{i}$

(b) For every maximal ideal $I$ in $A, A / I$ is a central simple algebra of dimension $i^{2}$ over its center.

Proof. [16, II.3.5].

Recall that, for $A \leq M_{i}$, the Formanek center $F(A)$ of $A$ is the subset of $A$ obtained by evaluating all central polynomials (for $i \times i$ matrices) without constant term, in any number of indeterminates; see [9.VIII].

\subsection{Proposition}

(a) $F(A)$ is an ideal in $Z(A)$, the center of $A$.

(b) $F(A)=Z(A)$ if and only if $A$ is an Azumaya algebra of rank $i^{2}$ over its center.

$$
\text { Proof. }[10,5.1,5.4]
$$

Let $n \geq 2, i \geq 2$ be two integers. Let $R$ be the algebra of $n$ generic $i \times i$ matrices (see Introduction, or [11]).

Let $B$ be the algebra of invariants (for $n$-tuples of $i \times i$ matrices), which we define by

$$
B=\left\{f: M_{i}^{n} \stackrel{\text { polyn. }}{\rightarrow} \mathbf{C}: f\left(m x m^{-1}\right)=f(x) \text { for all } x \in M_{i}^{n}, m \in \mathrm{GL}_{i}\right\}
$$


Then $S=B R$ is the algebra of concomitants (also known as the trace algebra), and

1.4. Proposition.

$$
\begin{aligned}
& S=\left\{f: M_{i}^{n} \stackrel{\text { polyn. }}{\rightarrow} M_{i}: f\left(m x m^{-1}\right)=m f(x) m^{-1}\right. \\
& \left.\qquad \text { for all } x \in M_{i}^{n}, m \in \mathrm{GL}_{i}\right\} .
\end{aligned}
$$

\section{Proof. [11, 2.1].}

Is it possible to describe $R$ by stronger intertwining conditions? The definition of Taylor's $\mathscr{D}(\Omega)$ algebra suggests that this might be possible, if we accept noninvertible intertwining matrices. Unfortunately, this works only for $n=i=2$, where we have the

\subsection{Proposition. Let}

$$
\begin{aligned}
R^{\prime}= & \left\{f: M_{2}^{2} \stackrel{\text { polyn. }}{\rightarrow} M_{2}, f(x) m=m f(y)\right. \\
& \text { whenever } \left.x, y \in M_{2}^{2}, m \in M_{2} \text { and } x m=m y\right\} .
\end{aligned}
$$

Then $R^{\prime}=R$, the algebra of 2 generic $2 \times 2$ matrices.

Proof. Basic structure results for $R$ are given in [7]. By (1.4),

$$
\begin{aligned}
S=\left\{f: M_{2}^{2} \stackrel{\text { polyn. }}{\rightarrow} M_{2}, f(x) m=m f(y)\right. \\
\left.\quad \text { when } x, y \in M_{2}^{2}, m \in \mathrm{GL}_{2} \text { and } x m=m y\right\}
\end{aligned}
$$

and thus $R \subseteq R^{\prime} \subseteq S$. Now $R$ and $S$ have the same commutator ideal $K$ [7], so $R^{\prime}$ will also have $K$ as its commutator ideal, and $R / K \subseteq R^{\prime} / K \subseteq$ $S / K$. Let $\bar{X}_{1}, \bar{X}_{2}, \bar{T}_{1}, \bar{T}_{2}$ be the classes $\bmod K$ of $X_{1}, X_{2}, \operatorname{tr} X_{1}, \operatorname{tr} X_{2} \in S$. Then $R / K \simeq \mathrm{C}\left[\bar{X}_{1}, \bar{X}_{2}\right]$ and $S / K \simeq \mathrm{C}\left[\bar{X}_{1}, \bar{X}_{2}, \bar{T}_{1}, \bar{T}_{2}\right]$. Take $a_{1}, b_{1}, c_{1}, a_{2}, b_{2}, c_{2} \in \mathbf{C}$ and let

$$
\begin{aligned}
x_{1}=\left(\begin{array}{lc}
a_{1} & 0 \\
0 & b_{1}-a_{1}
\end{array}\right) & x_{2}=\left(\begin{array}{lc}
a_{2} & 0 \\
0 & b_{2}-a_{2}
\end{array}\right) \\
y_{1}=\left(\begin{array}{cc}
a_{1} & 0 \\
0 & c_{1}-a_{1}
\end{array}\right) & y_{2}=\left(\begin{array}{lc}
a_{2} & 0 \\
0 & c_{2}-a_{2}
\end{array}\right) .
\end{aligned}
$$


Let $x=\left(x_{1}, x_{2}\right)$ and $y=\left(y_{1}, y_{2}\right)$. Note that $x\left(\begin{array}{ll}1 & 0 \\ 0 & 0\end{array}\right)=\left(\begin{array}{ll}1 & 0 \\ 0 & 0\end{array}\right) y$. Let $m=$ $\left(\begin{array}{ll}1 & 0 \\ 0 & 0\end{array}\right)$. Suppose $f \in S / K$. Write $f=\sum \lambda_{i j k l} \bar{X}_{1}^{i} \bar{X}_{2}^{j} \bar{T}_{2}^{k} \bar{T}_{2}^{l}$ (where $\lambda_{i j k l} \in \mathbf{C}$ and the sum is finite). If $f \in R^{\prime} / K, f(x) m=m f(y)$, which forces $\lambda_{i j k l}=$ 0 when $k \neq 0$ or $l \neq 0$. Thus $f \in R^{\prime} / K$ implies that $f=\sum \lambda_{i j 00} \bar{X}_{1}^{i} \bar{X}_{2}^{j} \in$ $R / K$, and from $R^{\prime} / K=R / K$, it follows that $R^{\prime}=R$ (the final part of this argument is due to E. Formanek).

From this description of $R^{\prime}=R$, we obtain

$$
Z(R)=Z\left(R^{\prime}\right)=\left\{f: M_{2}^{2} \stackrel{\text { polyn. }}{\rightarrow} \mathbf{C}, f(x)=f(y)\right.
$$

$$
\text { whenever } \left.x, y \in M_{2}^{2}, m \neq 0 \text {, and } x m=m y\right\}
$$

from which we can get

\subsection{Corollary.}

$$
Z(R)=\mathrm{C}+B\left(X_{1} X_{2}-X_{2} X_{1}\right)^{2} .
$$

This appears in [7] with a different proof.

However, results analogous to (1.5) fail for $n>2$ or $i>2$.

1.7. EXAMPLE (Formanek). Let $R$ be the algebra of 3 generic $2 \times 2$ matrices. Consider $f \in S, f\left(X_{1}, X_{2}, X_{3}\right)=\operatorname{tr} X_{1}\left(X_{2} X_{3}-X_{3} X_{2}\right)$; then $f$ satisfies the strong intertwining condition, but $f \notin R$. Similar examples can be given for pairs of $i \times i$ matrices $(i>2)$, and of course for $n$-tuples of $i \times i$ matrices $(i, n>2)$.

We now go back to the algebra of invariants $B$ ( $n, i$ arbitrary). $B$ is a finitely generated algebra; more precisely

1.8. Proposition. $B$ is generated by $\left\{\operatorname{tr}\left(X_{q_{1}} \cdots X_{q_{r}}\right): r \leq 2^{i}-1\right\}$.

Proof. [11, 1.3].

It follows that $B$ is Noetherian.

Fix a set of generators $z_{1}, \ldots, z_{p}$ for $B$. This defines a map $\Pi$ : $M_{i}^{n} \rightarrow \mathbf{C}^{p}$.

1.9. Definition. Let $\hat{M}_{i}^{n}=\left\{\left(x_{1}, \ldots, x_{n}\right) \in M_{i}^{n}: x_{1}, \ldots, x_{n}\right.$ generate $\left.M_{\imath}\right\}$. Let $\check{M}_{i}^{n}=M_{i}^{n}-\hat{M}_{i}^{n}$. 
Note that $\hat{M}_{i}^{n}$ is open and dense in $M_{i}^{n}$.

Two important facts are contained in

1.10. Proposition. (a) $\Pi$ maps $M_{i}^{n}$ onto an affine algebraic subvariety $\Delta$ in $\mathbf{C}^{p}, \operatorname{dim} \Delta=(n-1) i^{2}+1$.

(b) $\Pi$ restricted to $\hat{M}_{i}^{n}$ maps onto an open, smooth subset $\hat{\Delta}$ of $\Delta$; in fact, $\hat{M}_{i}^{n}$ is the total space of a principal bundle with fiber $\mathrm{PGL}_{i}$ and base $\hat{\Delta}$.

Proof. [10, 4.1.5.10]

We now attack the spectral study of Banach PI-algebras. The fundamental structure result is

1.11. Proposition. Let $A$ be a primitive Banach algebra satisfying a polynomial identity of degree $d$.

Then $A \simeq M_{i}$ for some $i \leq[d / 2]$

Proof. By a theorem of Kaplansky [9, II.1], $A$ is a central simple algebra of dimension $i^{2}$ over its center $Z$, with $2 i \leq d$. By the GelfandMazur theorem, $Z \simeq \mathbf{C}$. Since $\mathbf{C}$ is algebraically closed, we conclude that $A \simeq M_{i}$.

1.12. Corollary. Assume $A$ is a unital Banach algebra that satisfies an identity of degree $d$, and let I be an ideal of $A$. Then (a), (b), (c) are equivalent and imply (d):
(a) I is maximal
(b) $I$ is primitive
(c) $A / I \simeq M_{i}(2 i \leq d)$
(d) I is closed.

Let $A$ be a unital Banach PI-algebra, and take $a=\left(a_{1}, \ldots, a_{n}\right) \in A^{n}$. All irreducible representations of $A$ are finite-dimensional, and this will allow us to define the joint spectrum of $\left(a_{1}, \ldots, a_{n}\right)$ as a subset of $\Omega=\bigcup_{i=1}^{\infty} M_{i}^{n}$.

\subsection{DeFINITION. Let}

$$
\operatorname{sp}_{i}(a)=\left\{\left(\varphi\left(a_{1}\right), \ldots, \varphi\left(a_{n}\right)\right): \varphi \text { irreducible representation } A \rightarrow M_{i}\right\}
$$

and $\operatorname{sp}(a)=\bigcup_{i=1}^{\infty} \operatorname{sp}_{i}(a)$. (Actually the union is always finite.)

We will need the notion of full spectrum. 
1.14. DeFINITION. Let

$$
\begin{aligned}
f \operatorname{sp}_{i}(a)=\left\{\left(\psi\left(a_{1}\right), \ldots, \psi\left(a_{n}\right)\right):\right. & \psi \text { nondegenerate } \\
& \text { representation } \left.A \rightarrow M_{i}\right\}
\end{aligned}
$$

and $f \operatorname{sp}(a)=\bigcup_{i=1}^{\infty} f \operatorname{sp}_{i}(a)$.

Also interesting is the polynomial spectrum.

\subsection{DeFINITION. Let}

$$
\begin{array}{r}
p \operatorname{sp}_{i}(a)=\left\{x=\left(x_{1}, \ldots, x_{n}\right) \in M_{i}^{n}: \rho p(x) \leq \rho p(a) \text { for all } p \in F_{n}\right\} \\
(\rho=\text { spectral radius }),
\end{array}
$$

and $p \operatorname{sp}(a)=\bigcup_{i=1}^{\infty} p \operatorname{sp}(a)$.

Clearly $\varnothing \subset \operatorname{sp}(a) \subset f \operatorname{sp}(a) \subseteq p \operatorname{sp}(a)$.

We will not consider the problem of the Gelfand transform (can we represent any semiprimitive Banach PI-algebra as an algebra of continuous matrix-valued functions?) in full generality here. Let us just mention the

1.16. Proposition (Fell). Let $A$ be a unital $C^{*}$-algebra such that $A / I \simeq M_{i}$ for all maximal ideals $I$ of $A$ ( $i$ is fixed). Let $Z$ be the center of $A$ : then $Z \simeq C(X)$ for some compact Hausdorff space $X$. There is a bundle $\mathscr{M}$ with fiber $M_{i}$ and base $X$ such that $A \simeq \Gamma(X, \mathscr{M})$, the algebra of all continuous sections of over $X$.

\section{Proof [6, Th. 3.2].}

Thus Azumaya $C^{*}$-algebras can be represented as section algebras (since the bundle $\mathscr{M}$ need not be trivial, we cannot always write $A=$ $\left.C\left(X, M_{i}\right)\right)$. A similar result will be given for some Azumaya algebras of holomorphic functions (4.19).

\section{Chapter Two. Free Algebras of Functions}

In his papers [14] and [15], Taylor studied "localizations" of the free algebra. We fix $n \geq 1$. An embedding $F_{n} \rightarrow \mathscr{A}$ (where $\mathscr{A}$ is a Fréchet algebra, i.e., a complete, metrizable, locally $m$-convex algebra) is said to be a localization if there exist $n$ linear maps $\Delta_{1}, \ldots, \Delta_{n}: \mathscr{A} \rightarrow \mathscr{A} \hat{\otimes} \mathscr{A}(\hat{\otimes}$ is the completed inductive tensor product) that have properties similar to partial differential quotients $\left[15\right.$, p. 6]. If, furthermore, $\Delta_{1}, \ldots, \Delta_{n}$ satisfy 
conditions analogous to the Cauchy estimates, $\mathscr{A}$ will be called a free analytic algebra [15, p. 13]. When the map $F_{n} \rightarrow \mathscr{A}$ is understood, we shall just say that $\mathscr{A}$ is a localization. Taylor gives many examples of localizations of $F_{1}$, the polynomials algebra in one indeterminate. He shows that $\mathscr{A}$ is a free analytic algebra (in one indeterminate) if and only if $\mathscr{A}=\mathscr{O}(U)$ for $U$ open in $\mathbf{C}$. Since the algebras $\mathcal{O}(U)$ give a one-variable functional calculus, we can hope that free analytic algebras (in $n$ indeterminates) will lead to a functional calculus for $n$ noncommuting variables in a Banach algebra.

We shall also need the notion of "lmc completion". The lmc completion $\tilde{\mathscr{A}}$ of a topological algebra $\mathscr{A}$ is the Hausdorff completion of $\mathscr{A}$ with respect to the family of all continuous submultiplicative seminorms on $\mathscr{A}$. It has the universal property:

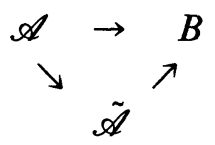

viz. every continuous homomorphism $\mathscr{A} \rightarrow B$ ( $B$ Banach algebra) factors through $\tilde{\mathscr{A}}$; see [13, p. 178] for an equivalent definition.

We assume henceforth $n \geq 2$. Let us investigate some "free algebras of functions".

Every free polynomial $p$ of degree $d$ in $F_{n}$ can be written as $p=\sum_{|\sigma|=0}^{d} \lambda_{\sigma} X^{\sigma}$ where $\sigma$ is a noncommutative multi-index, $|\sigma|$ its length, and $\lambda_{\sigma}$ is a complex coefficient. Let $\left.I=\right] 0 \infty$ ]. On $I^{n}$, we have the partial ordering $a=\left(a_{1}, \ldots, a_{n}\right)<b=\left(b_{1}, \ldots, b_{n}\right)$ if and only if $a_{1}<b_{1}, \ldots, a_{n}$ $<b_{n}$. Let $r \in I^{n}$. We introduce the algebra of power series [14], [15]:

\subsection{Definition.}

$$
\mathscr{F}(r)=\left\{\sum_{|\sigma|=0}^{\infty} \lambda_{\sigma} X^{\sigma}: \sum_{|\sigma|=0}^{\infty}\left|\lambda_{\sigma}\right| t^{\sigma}<\infty \text { for all } t \in I^{n}, t<r\right\} .
$$

Endowed with the obvious family of (semi)norms, $\mathscr{F}(r)$ is a Fréchet algebra, and a free analytic algebra [15, p. 17]. Let $\mathscr{F}(\infty)=\mathscr{F}(r)$ where $r=(\infty, \ldots, \infty) . \mathscr{F}(\infty)$ is the lmc completion of $F_{n}$, and can be regarded as the algebra of "free entire analytic functions" (although free power series do not correspond to functions in the sense of mappings). Taylor claimed [14, p. 235] that $\mathscr{F}(r)$ was never nuclear (as a Fréchet space). However, we have the

2.2. Proposition. Let $r=\left(r_{1}, \ldots, r_{n}\right) \in I^{n} . \mathscr{F}(r)$ is nuclear if and only if at most one of the $r_{1}, \ldots, r_{n}$ is finite. 
Proof. We follow [14]. $\mathscr{F}(r)$ is nuclear provided that, for every $\rho^{\prime} \in I^{n}, \rho^{\prime}<r$, there is $\rho \in I^{n}, \rho^{\prime}<\rho<r$, for which the series

$$
\sum_{k_{1}, \ldots, k_{n}} \frac{\left(k_{1}+\cdots+k_{n}\right) !}{k_{1} ! \cdots k_{n} !}\left(\frac{\rho_{1}^{\prime}}{\rho_{1}}\right)^{k_{1}} \cdots\left(\frac{\rho_{n}^{\prime}}{\rho_{n}}\right)^{k_{n}}
$$

is convergent. This series can be rewritten as

$$
\sum_{k=0}^{\infty}\left(\frac{\rho_{1}^{\prime}}{\rho_{1}}+\cdots+\frac{\rho_{n}^{\prime}}{\rho_{n}}\right)^{k} \quad\left(k=k_{1}+\cdots+k_{n}\right)
$$

which converges if

$$
\frac{\rho_{1}^{\prime}}{\rho_{1}}+\cdots+\frac{\rho_{n}^{\prime}}{\rho_{n}}<1
$$

If (and only if) at most one of the $r_{1}, \ldots, r_{n}$ is finite, then for every choice of $\rho^{\prime}<r$ we will be able to find $\rho, \rho^{\prime}<\rho<r$, such that the above inequality is satisfied, and $\mathscr{F}(r)$ will be nuclear.

\subsection{Corollary. $\mathscr{F}(\infty)$ is nuclear.}

Here is the relationship between free power series and the functional calculus problem.

2.4. Proposition. Let $A$ be a unitial Banach algebra, and take $a_{1}, \ldots, a_{n} \in A$. Choose $r_{1}>\left\|a_{1}\right\|, \ldots, r_{n}>\left\|a_{n}\right\|$. There is a continuous algebra homomorphism $\mathscr{F}(r) \rightarrow A$ that maps $1 \mapsto 1, X_{1} \mapsto a_{1}, \ldots, X_{n} \mapsto a_{n}$.

\section{Proof. Elementary.}

But, as we observed earlier, $\mathscr{F}(r)$ can hardly be regarded as an algebra of functions. Therefore, we shall now turn our attention to a quite different kind of algebra, based on the idea of function rather than power series.

The following definitions are borrowed from [14, p. 238]. Let $\Omega=$ $\bigcup_{i=1}^{\infty} M_{i}^{n}$ (topological disjoint union). If $x=\left(x_{1}, \ldots, x_{n}\right) \in M_{i}^{n}, y=$ $\left(y_{1}, \ldots, y_{n}\right) \in M_{j}^{n}, x \oplus y$ will denote

$$
\left(\begin{array}{ll}
x & 0 \\
0 & y
\end{array}\right)=\left(\left(\begin{array}{cc}
x_{1} & 0 \\
0 & y_{1}
\end{array}\right), \ldots,\left(\begin{array}{cc}
x_{n} & 0 \\
0 & y_{n}
\end{array}\right)\right) \in M_{i+j}^{n} .
$$

Let $U$ be an open subset of $\Omega$, let $U_{i}=U \cap M_{i}^{n}$. Then $U_{i}$ is open in $M_{i}$. 
We denote the Fréchet algebra of holomorphic functions in $U_{\iota}$ with values in $M_{\imath}$ by $\mathcal{O}\left(U_{i}, M_{i}\right)$.

Let $\mathscr{B}(U)=\prod_{i=1}^{\infty} \mathcal{O}\left(U_{i}, M_{i}\right)$ be the topological direct product. Thus an element $f \in \mathscr{B}(U)$ is a function on $U$ which, when restricted to $U_{l}$, is a holomorphic function with values in $M_{l}$.

Note that $\mathscr{B}(U)$ is a nuclear Fréchet algebra. We shall say that $U$ open in $\Omega$ is normal if $x \in U_{i}, y \in U_{j}$ implies $x \oplus y \in U_{t+j}$.

2.5. Definition. Suppose $U$ is a nonempty open normal subset of $\Omega$. Then

$$
\begin{aligned}
\mathscr{D}(U)= & \{f \in \mathscr{B}(U): f(x) m=m f(y) \\
& \text { whenever } \left.x \in U_{l}, y \in U_{j}, m \in M_{l}, \text { and } x m=m y\right\} .
\end{aligned}
$$

$\mathscr{D}(U)$ is a closed subalgebra of $\mathscr{B}(U)$ and hence is also a nuclear Fréchet algebra. We have a natural map $F_{n} \rightarrow \mathscr{D}(U)$. As no (nonzero) free polynomial vanishes in all matrix algebras, this map is injective. Here is a crucial property.

2.6. Proposition. If $U$ is a nonempty open normal subset of $\Omega, \mathscr{D}(U)$ is a projective limit of Banach PI-algebra.

Proof. $\mathscr{D}(U)$ is a closed subalgebra of $\mathscr{B}(U)$. Any continuous seminorm $\nu$ on $\mathscr{D}(U)$ is essentially given by $\nu(f)=\max \{\|f(x)\|: x \in K\}$ for some $K$ compact in $U$, so $K \subset \bigcup_{j=1}^{i} M_{j}^{n}$. Thus $\nu^{-1}(0)$ contain all polynomial identities of $i \times i$ matrices, and the normed algebra $\mathscr{D}(U) / \nu^{-1}(0)$ $\leq M_{i}$. Taking an increasing sequence of compact sets and completing, we can realize $\mathscr{D}(U)$ as a projective limit of Banach PI-algebras.

2.7. Corollary. Let $A$ be a Banach algebra, and $\varphi: \mathscr{D}(U) \rightarrow A$ a continuous homomorphism. There exists $i \in \mathbf{N}$ such that $\varphi \mathscr{D}(U) \leq M_{\imath}$ (hence $\overline{\varphi \mathscr{D}(U)} \leq M_{i}$ ).

Thus, if $A$ is a Banach algebra with unit, and $a_{1}, \ldots, a_{n} \in A$, we can hope to get a functional calculus map $\mathscr{D}(U) \rightarrow A, \quad 1 \mapsto 1, \quad X_{1} \mapsto$ $a_{1}, \ldots, X_{n} \mapsto a_{n}$ only when the subalgebra of $A$ generated by $a_{1}, \ldots, a_{n}$ satisfies some identity, hence all identities of $i \times i$ matrices for some $i$ (1.1).

Since $\mathscr{D}(U)$ is $1 \mathrm{mc}$ and complete, the embedding $F_{n} \rightarrow \mathscr{D}(U)$ yields an injection $\mathscr{F}(\infty) \rightarrow \mathscr{D}(U)$. In particular, $\mathscr{F}(\infty) \rightarrow \mathscr{D}(\Omega)$. We know that $\mathscr{F}(\infty)$ is an algebra of "free entire power series", while $\mathscr{D}(\Omega)$ is an algebra of "entire matrix-valued functions". 
2.8. Proposition. The map $\mathscr{F}(\infty) \rightarrow \mathscr{D}(\Omega)$ is not surjective.

Proof. If it were, $\mathscr{F}(\infty)$ and $\mathscr{D}(\Omega)$ would be isomorphic Fréchet spaces; but on $\mathscr{D}(\Omega)$ there is no continuous norm $(2.7)$ and $\mathscr{F}(\infty)$ admits many continuous norms.

A more concrete explanation is the following (see Introduction).

2.9. EXAMPLE. Let $n=2$, consider

$$
f\left(X_{1}, X_{2}\right)=\sum_{k=2}^{\infty} S_{k}\left(X_{1}, X_{1} X_{2}, \ldots, X_{1} X_{2}^{k-1}\right) .
$$

Then $f \notin \mathscr{F}(\infty)$ : write $f$ as a power series $\sum \lambda_{\sigma} X^{\sigma}$, and observe that for all $d \in \mathbf{N}$, there is a multi-index $\sigma,|\sigma| \geq d, \lambda_{\sigma}=1$; thus $\sum \lambda_{\sigma} X^{\sigma}$ cannot have infinite radius of convergence.

But $f \in \mathscr{D}(\Omega)$ : if $x=\left(x_{1}, x_{2}\right) \in M_{i}^{2}, f\left(x_{1}, x_{2}\right)$ is given by the finite sum $\sum_{k=2}^{2 i} S_{k}\left(x_{1}, x_{1} x_{2}, \ldots, x_{1} x_{2}^{k-1}\right)$, and $f \in \mathscr{B}(\Omega)$; it is easily checked that $f \in \mathscr{D}(\Omega)$.

2.10. Proposition. Let $f \in \mathscr{D}(\Omega), x \in U_{i}, y \in U_{j}$. There is a linear map $\Delta: M_{i j}^{n} \rightarrow M_{i j}, z \mapsto f(x, y, z)$ such that

$$
f\left(\begin{array}{cc}
x & z \\
0 & y
\end{array}\right)=\left(\begin{array}{cc}
f(x) & \Delta f(x, y, z) \\
0 & f(y)
\end{array}\right)
$$

whenever $\left(\begin{array}{ll}x & z \\ 0 & y\end{array}\right) \in U_{i+j}$.

Proof. See [14, p. 239]. Note that $\left(\begin{array}{ll}x & 0 \\ 0 & y\end{array}\right) \in U_{i+j}$, and as $U$ is open, $\left(\begin{array}{ll}x & z \\ 0 & y\end{array}\right) \in U_{i+j}$ for all $z$ in a neighborhood of 0 in $M_{l j}^{n}$.

2.11. Proposition. Every $f \in \mathscr{D}(\Omega)$ has a formal free power series in $X_{1}, \ldots, X_{n}$.

Proof. For a given $i, f$ will have a power series as a function $M_{i}^{n}=\mathbf{C}^{n i^{2}} \rightarrow \mathbf{C}^{i^{2}}=M_{i}$. This Taylor series can be written as a power series in $X_{1}, \ldots, X_{n}$, say $\sum_{|\sigma|=0}^{\infty} \lambda_{\sigma}^{(i)} X^{\sigma}\left[15\right.$, p. 20]. As $M_{i}$ does not satisfy any polynomial identity of degree $<2 i$, the $\lambda_{\sigma}^{(i)}$ are uniquely determined for $|\sigma|<2 i$. We define $\lambda_{\sigma}=\lambda_{\sigma}^{(i)}$ for any $i>|\sigma| / 2$, and $\sum \lambda_{\sigma} X^{\sigma}$ is the formal power series for $f$. Example (2.9) shows that we lose control on the growth of the coefficients. 
Taylor proves $[14$, p. 242] that $\mathscr{D}(U)$ is a localization. However, it is not a free analytic algebra. Let us only show

2.12. Proposition. $\mathscr{D}(\Omega)$ is not a free analytic algebra.

Proof. Let $E$ be an infinite-dimensional Banach space. If $\mathscr{D}(\Omega)$ were a free analytic algebra, the set

$$
\begin{aligned}
V=\left\{\left(x_{1}, \ldots, x_{n}\right) \in(\mathscr{L}(E))^{n}:\right. \text { there is a continuous homomorphism } \\
\left.\mathscr{D}(\Omega) \rightarrow \mathscr{L}(E), 1 \mapsto 1, X_{1} \mapsto x_{1}, \ldots, X_{n} \mapsto x_{n}\right\}
\end{aligned}
$$

would be open in the norm topology of $(\mathscr{L}(E))^{n}[15$, p. 14]. But (2.7)

$$
V \subseteq W=\left\{\left(x_{1}, \ldots, x_{n}\right) \in(\mathscr{L}(E))^{n}: x_{1}, \ldots, x_{n}\right.
$$

generate a PI-subalgebra of $\mathscr{L}(E)\}$.

Since $0=(0, \ldots, 0) \in W$ and $W$ is not a neighborhood of $0, V$ cannot be open. A similar argument shows that $\mathscr{D}(U)$ is not a free analytic algebra.

In the setting of matrix-valued functions, we can study envelopes and domains of holomorphy.

As usual, let $U$ be a nonempty, open normal subset of $\Omega$. By a representation $\mathscr{D}(U) \rightarrow M_{i}$, we mean a continuous unital algebra homomorphism. Since $\mathscr{D}(U)$ is a localization of $F_{n}$, we have the

2.13. Proposition. Let $\left(x_{1}, \ldots, x_{n}\right) \in M_{i}^{n}$. There is at most one representation $\mathscr{D}(U) \rightarrow M_{i}, X_{1} \mapsto x_{1}, \ldots, X_{n} \mapsto x_{n}$.

Proof. [15, p. 7].

Of course, if $x=\left(x_{1}, \ldots, x_{n}\right) \in U_{i}$, evaluation at $x$ will give a representation. A natural question is: are there (finite-dimensional) representations which are not evaluations?

2.14. Definition. For $i=1,2,3, \ldots$, we let

$$
\begin{array}{r}
\tilde{U}_{i}=\left\{x \in M_{i}^{n}: \text { there is one representation } \mathscr{D}(U) \rightarrow M_{i},\right. \\
\left.X_{1} \mapsto x_{1}, \ldots, X_{n} \mapsto x_{n}\right\} .
\end{array}
$$

Then $\tilde{U}=\bigcup_{i=1}^{\infty} \tilde{U}_{i}$ is the envelope of holomorphy of $U$. Since $U_{i} \subseteq \tilde{U}_{i} \subseteq$ $M_{i}^{n}$, we have $U \subseteq \tilde{U} \subseteq \Omega$; thus we do not have to consider "Riemann domains". 
2.15. Conjecture. We believe that $\tilde{U}$ is always open in $\Omega$. If it were true that $\mathscr{D}(U)$ is a free analytic algebra, this would follow from [15]. Note that $U$ is normal; if $U$ is open, we have a natural isomorphism $\mathscr{D}(\tilde{U}) \rightarrow \mathscr{D}(U)$.

2.16. Definition. $U$ is a domain of holomorphy if $\tilde{U}=U$.

Although we do not have a complete characterization for domains of holomorphy, we have necessary conditions contained in the

2.17. Proposition. Let $U$ be a domain of holomorphy. Then:

(a) For all $i, U_{i}$ is holomorphically convex (in the classical sense, viz. as a subset of $\mathbf{C}^{n i^{2}}$ ).

(b) If $x \in U_{i}$ and $m \in \mathrm{GL}_{i}$, then $\mathrm{mxm}^{-1} \in U_{i}$

(c) If $x \in U_{i}$ and $y \in U_{j}$, then

$$
\left(\begin{array}{ll}
x & z \\
0 & y
\end{array}\right) \in U_{i+j} \quad \text { for all } z \in M_{i j}^{n} .
$$

(d) If $\left(\begin{array}{ll}x & 2 \\ 0 & y\end{array}\right) \in U_{i+j}$, then $x \in U_{i}$ and $y \in U_{j}$.

Proof. (a) Assume $U_{i}$ is not a domain of holomorphy in $\mathbf{C}^{n i^{2}}$. Let $\hat{U}_{i}$ be its classical envelope of holomorphy, which is a manifold spread over $\mathbf{C}^{n i^{2}}$. Every holomorphic function $g: U_{i} \rightarrow M_{i}$ extends to $\hat{g}: \hat{U}_{i} \rightarrow M_{i}$. Take $x \in \hat{U}_{i}$, let $\psi: \mathscr{D}(U) \rightarrow M_{i}, f \mapsto \hat{g}(x)$ (where $\left.f\right|_{U_{i}}=g$ ); this defines a representation of $\mathscr{D}(U)$. If $x$ does not lie over $U_{i}, \psi$ cannot be given by any point of $U_{i}$, so $U$ is not a domain of holomorphy.

(b) $\psi(f)=m f(x)^{-1}$ defines a representation, which must be given by evaluation at $m \times m^{-1}$.

(c) As $U$ is normal, $x \in U_{i}$ and $y \in U_{j}$ imply that

$$
\left(\begin{array}{ll}
x & 0 \\
0 & y
\end{array}\right) \in U_{i+j}
$$

As $U$ is open, for $w \in M_{i j}^{n}$, w small enough,

$$
\left(\begin{array}{ll}
x & w \\
0 & y
\end{array}\right) \in U_{i+j}
$$

Now, by (b),

$$
\left(\begin{array}{ll}
t & 0 \\
0 & 1
\end{array}\right)\left(\begin{array}{ll}
x & w \\
0 & y
\end{array}\right)\left(\begin{array}{ll}
t^{-1} & 0 \\
0 & 1
\end{array}\right)=\left(\begin{array}{cc}
x & t w \\
0 & y
\end{array}\right) \in U_{i+j}
$$

for all $t \in \mathbf{C}^{*}$. Since $t w$ is arbitrary, we see that

$$
\left.\left(\begin{array}{ll}
x & z \\
0 & y
\end{array}\right) \in U_{i+j} \quad \text { for all } z \in M_{i j}^{n} \text { (see also }(2.10)\right) \text {. }
$$


(d) This follows from [15, p. 7].

We do not know if these four conditions are sufficient, i.e., if every normal, open $U$ that satisfies (a), (b), (c), (d) is a domain of holomorphy.

We also have a class of examples, the holomorphic polyhedra. Let $V$ be a domain of holomorphy (e.g., $\Omega$ ), take $f_{1}, \ldots, f_{q} \in \mathscr{D}(V)$, and consider

$$
U=\left\{x \in V: \rho f_{1}(x)<1, \ldots, \rho f_{q}(x)<1\right\} .
$$

$U$ is open and normal, moreover

\subsection{Proposition. $U$ is a domain of holomorphy.}

Proof. First observe that $U \subseteq V \Rightarrow U \subseteq \tilde{U} \subseteq \tilde{V}=V$. Suppose $x \in \tilde{U}$ - $U$. We can assume, without loss of generality, that $\rho f_{1}(x) \geq 1$, so there is $\eta \in \mathbf{C},|\eta| \geq 1$, such that $f_{1}(x)-\eta$ is not invertible in $M_{i}$. But $f_{1}-\eta$ is invertible in $\mathscr{D}(U)$, and a unital representation should map invertibles onto invertibles.

In classical complex analysis, domains of holomorphy are holomorphically convex, and conversely (Cartan-Thullen). Here, the $\mathscr{D}(U)$-convex hull of a compact subset of $U$ will almost never be compact, even if $U$ is a domain of holomorphy. Still, we think that domains of holomorphy should be "limits" of holomorphic polyhedra.

\section{Chapter Three. PI-Algebras of Functions}

Let $A$ be a unital Banach algebra, and $a_{1}, \ldots, a_{n} \in A$. We have shown that a functional calculus map $\mathscr{D}(U) \rightarrow A$ could exist only if the subalgebra of $\boldsymbol{A}$ generated by $a_{1}, \ldots, a_{n}$ satisfied a polynomial identity, hence all identities of $i \times i$ matrices for some $i$ (2.7).

Without loss of generality, we shall assume that $A$ itself satisfies the identities of $i \times i$ matrices, i.e., $A \leq M_{i}$.

Suppose there exists $\varphi: \mathscr{D}(\Omega) \rightarrow A, 1 \mapsto 1, X_{1} \mapsto a_{1}, \ldots, X_{n} \mapsto a_{n} . \varphi$ will then vanish on the ideal of polynomial identities of $i \times i$ matrices, hence on its closure $I_{i}=\left\{f \in \mathscr{D}(\Omega): f=0\right.$ on $\left.M_{i}^{n}\right\}$ (actually $f=0$ on $M_{i}^{n}$ implies $f=0$ on $\left.M_{j}^{n}, j \leq i\right)$. Thus $\varphi$ will yield a map $\mathscr{D}(\Omega) / I_{i} \rightarrow A$. The quotient $\mathscr{D}(\Omega) / I_{i}$ can be expressed as an algebra of functions taking $n$-tuples of $j \times j$ matrices $(1 \leq j \leq i)$ to $j \times j$ matrices, and $\mathscr{D}(\Omega) / I_{i} \leq$ $M_{i}$. 
In order to attack the question of the existence of $\varphi$, we need a satisfactory theory of such quotient algebras, more generally of quotients $\mathscr{D}(U) /\left\{f \in \mathscr{D}(U): f\right.$ vanishes on $U_{j}$ for all $\left.j \leq i\right\}$, where $U$ is open and normal in $\Omega$. Therefore, we are led to investigate PI-algebras of functions, similar to the $\mathscr{D}(U)$, but now $U \subseteq \bigcup_{j=1}^{i} M_{j}^{n}$ has only finitely many "levels".

We shall make a simplifying assumption, viz. consider just $U \subseteq M_{i}^{n}$ ( $U$ has only one level). In the two cases we will study later, this is no loss of generality:

- If $i=2$, we can always embed ( $n$-tuples of) scalars as ( $n$-tuples of) scalar matrices (Chapter Five)

- When our PI-algebra of functions turns out to be Azumaya of rank $i^{2}, U$ must be contained in $M_{i}^{n}$ (Chapter Four).

Another argument for restricting ourselves to "one-level" algebras is that we can embed all $M_{j}, 1 \leq j \leq i$, in $M_{i !}$ (see also 6.6)

Let us thus take $U$ open in $M_{i}^{n}$.

\subsection{DeFinition.}

$$
\begin{aligned}
& \mathscr{H}(U)=\left\{f: U \stackrel{\text { holom. }}{\rightarrow} M_{i}, f(x) m=m f(y)\right. \text { whenever } \\
& \left.\qquad x, y \in U, m \in M_{i}, x m=m y\right\} .
\end{aligned}
$$

3.2. Definition. $\mathscr{R}(U)=$ the closure in $\mathcal{O}\left(U, M_{i}\right)$ of the algebra $R$ of $n$ generic $i \times i$ matrices.

Clearly $\mathscr{R}(U) \subseteq \mathscr{H}(U)$. In some sense, $\mathscr{R}(U)$ is the smallest algebra we can define, if we demand that it be closed in $\mathcal{O}\left(U, M_{i}\right)$ and contain the generic matrices. We also expect every function of $n i \times i$ matrices to satisfy the intertwining condition in (3.1), so $\mathscr{H}(U)$ is the largest algebra we can reasonably work with.

Neither of these two definitions is totally satisfying, (3.1) in view of example (1.7), (3.2) because we do not expect all domains $U$ to be Runge. But both will prove to be useful in the sequel. In the next two chapters, we shall show, under suitable assumptions on $U$, that $\mathscr{R}(U)=\mathscr{H}(U)$. Let us already mention

\subsection{Proposition. $\mathscr{R}\left(M_{2}^{2}\right)=\mathscr{H}\left(M_{2}^{2}\right)$.}

Proof. Let $f \in \mathscr{H}\left(M_{2}^{2}\right) ; f$ is the sum of its Taylor series (as $f$ : $\mathbf{C}^{8} \rightarrow M_{2}$ ), i.e., $f=\sum_{|\mu|=0}^{\infty} c_{\mu} t^{\mu}$ where $c_{\mu} \in M_{2}, \mu$ is a commutative multi- 
index, and $t$ is a 8-tuple of complex numbers (the 8 entries of the matrices $X_{1}$ and $X_{2}$ ). Since the intertwining condition (3.1) is linear, $f_{k}=$ $\sum_{|\mu|=0}^{k} c_{\mu} t^{\mu} \in R$ (the algebra of 2 generic $2 \times 2$ matrices) by (1.5), and $f_{k} \stackrel{k \rightarrow \infty}{\rightarrow} f$, so $f \in \mathscr{R}\left(M_{2}^{2}\right)$. The reverse inclusion is obvious.

This result will be generalized later (4.17). Note that the analog of (3.3) fails when $n>2$ or $i>2$, because of (1.7).

\section{Chapter Four. The Implicit Function Theorem}

Algebras of holomorphic functions on polynomially convex sets in $\mathbf{C}^{n}$ were studied by H. Cartan and K. Oka in the 1940's. They yield one road (Waelbroeck) to the commutative functional calculus [8], while another approach (Shilov, Arens-Calderon) uses the Weil integral formula. We do not see any generalization of the Weil formula to functions of several noncommuting variables. On the other hand, Taylor [15, p. 23] proved an "implicit function theorem" that will play the role of the Oka-Cartan results.

4.1. Proposition. Let $\mathscr{F}=\mathscr{F}\left(r_{1}, \ldots, r_{n}, s_{1}, \ldots, s_{q}\right)$ be a power series algebra (2.1) in the indeterminates $X_{1}, \ldots, X_{n}, Y_{1}, \ldots, Y_{q}$. Let $p_{1}, \ldots, p_{q}$ be free polynomials in $X_{1}, \ldots, X_{n}$ and let $I$ be the closed ideal generated in $\mathscr{F}$ by $p_{1}-Y_{1}, \ldots, p_{q}-Y_{q}$.

Then $\mathscr{E}=\mathscr{F} / I$ is a free analytic algebra in the indeterminates $X_{1}, \ldots, X_{n}$, provided $I \neq \mathscr{F}$ (we do not distinguish between $X_{k}$ in $\mathscr{F}$ and its class $\bmod I, k=1, \ldots, n)$.

\section{Proof. [15, p. 26 (Example 3)].}

Recall that algebras of power series do map into Banach algebras (2.4), but not all functions in $\mathscr{D}(\Omega)$ have convergent power series expressions (2.9). However, this very example (2.9) indicates that power series algebras and matricial function algebras might coincide after factoring out the identities of $i \times i$ matrices. Our goal is to prove this, under suitable hypotheses. The idea is to map quotients of power series algebras into PI-algebra of functions (Chapter Three) by a "Gelfand transformation" and show that the homomorphism must be an isomorphism.

We want to map $\mathscr{E}=\mathscr{F} / I$ (4.1) into an algebra $\mathscr{H}(U)(3.1)$. Let us study $\mathscr{E}$ by looking at its finite-dimensional representations (a representation is always assumed to be continuous and nondegenerate). 
4.2. Definition. For $i=1,2,3, \ldots$, we let

$$
\begin{aligned}
U_{i}=\{x= & \left(x_{1}, \ldots, x_{n}\right) \in M_{i}^{n}: \text { there is a (unique, 2.13) } \\
& \text { representation } \left.\mathscr{E} \rightarrow M_{l}, X_{1} \mapsto x_{1}, \ldots, X_{n} \mapsto x_{n}\right\} .
\end{aligned}
$$

Note that each $U_{i}$ is open in $M_{i}^{n}[15, \mathrm{p} .14]$ and invariant under conjugation. What does $U_{i}$ look like? Can it be defined by analytic inequalities?

4.3. Proposition. Let \|\| be any algebra norm on $M_{i}$. If $x=$ $\left(x_{1}, \ldots, x_{n}\right) \in M_{i}^{n}$, and $\left\|x_{1}\right\|<r_{1}, \ldots,\left\|x_{n}\right\|<r_{n},\left\|p_{1}(x)\right\|<$ $s_{1}, \ldots,\left\|p_{q}(x)\right\|<s_{q}$, then $x \in U_{i}$.

Proof. This follows from (2.4)

We have a converse result

4.4. Proposition. If $x=\left(x_{1}, \ldots, x_{n}\right) \in U_{i}$, then $\rho\left(x_{1}\right)<$ $r_{1}, \ldots, \rho\left(x_{n}\right)<r_{n}, \rho p_{1}(x)<s_{1}, \ldots, \rho p_{q}(x)<s_{q}$.

Proof. Suppose $x \in U_{i}$. Let $\alpha$ be the corresponding representation $\mathscr{E} \rightarrow M_{i}$, and $\bar{\alpha}$ its lift $\mathscr{F} \rightarrow M_{i}$, so $\bar{\alpha}\left(X_{1}\right)=x_{1}$. Now $f=\sum_{k=0}^{\infty} c_{k} X_{1}^{k} \in \mathscr{F}$ if $\sum_{k=0}^{\infty} c_{k} t_{1}^{k}$ converges for all $t_{1} \in \mathbf{R}_{+}, t_{1}<r_{1}$, and then $\bar{\alpha}(f)=\sum_{k=0}^{\infty} c_{k} x_{1}^{k}$ converges only if $\rho\left(x_{1}\right) \leq r_{1}$. As $U_{i}$ is open we must have $\rho\left(x_{1}\right)<r_{1}$. Similarly, we can consider power series of a single variable, and show $\rho\left(x_{2}\right)<r_{2}, \ldots, \rho\left(x_{n}\right)<r_{n}, \rho p_{1}(x)<s_{1}, \ldots, \rho p_{q}(x)<s_{q}$.

4.5. Remark. Combining (4.3) and (4.4), we see that, for $x \in M_{i}^{n}$,

$$
\left.\begin{array}{l}
\left\|x_{1}\right\|<r_{1}, \ldots,\left\|x_{n}\right\|<r_{n} \\
\left\|p_{1}(x)\right\|<s_{1}, \ldots,\left\|p_{q}(x)\right\|<s_{q}
\end{array}\right\} \Rightarrow x \in U_{i} \Rightarrow\left\{\begin{array}{l}
\rho\left(x_{1}\right)<r_{1}, \ldots, \rho\left(x_{n}\right)<r \\
\rho p_{1}(x)<r_{1}, \ldots, \rho p_{q}(x)<s_{q}
\end{array}\right.
$$

which does not completely determine $U_{i}$ (except when $i=1$ ), but does give some control on it. Later, projective limit arguments and the Gelfand-Beurling formula will allow us to obtain functional calculi on domains defined by analytic inequalities (6.4).

4.6. REMARK. If (for example) $p_{1}$ is a central polynomial for $i \times i$ matrices, then $p_{1}(x)$ is a scalar matrix for all $x \in M_{i}^{n},\left\|p_{1}(x)\right\|=\rho p_{1}(x)$, and the condition for $p_{1}$ is completely known. 
4.7. REMARK. With results like (2.18) in mind, one could believe that actually (4.4) determines $U_{i}$, i.e.,

$$
\begin{aligned}
U_{i}=\left\{x \in M_{i}^{n}: \rho\left(x_{1}\right)\right. & <r_{1}, \ldots, \rho\left(x_{n}\right)<r_{n}, \\
& \left.\rho p_{1}(x)<s_{1}, \ldots, \rho p_{q}(x)<s_{q}\right\} .
\end{aligned}
$$

This is not the case; let $n=2, q=0$ (so $\mathscr{E}=\mathscr{F}$ ), $r_{1}=r_{2}=1, i=2$. Then

$$
U_{2} \subset\left\{\left(x_{1}, x_{2}\right): \rho\left(x_{1}\right)<1, \rho\left(x_{2}\right)<1\right\}
$$

and inclusion is strict: let $f\left(X_{1}, X_{2}\right)=\sum_{k=0}^{\infty}\left(X_{1} X_{2}\right)^{k}, f \in \mathscr{F}$, and $\left(x_{1}, x_{2}\right)$ $\in U_{2}$ implies $\rho\left(x_{1} x_{2}\right)<1$, which does not follow from $\rho\left(x_{1}\right)<1, \rho\left(x_{2}\right)$ $<1$.

Now let $i$ be chosen such that $U_{i} \neq \varnothing$, but $U_{j}=\varnothing$ for $j<i$. Note that all we are going to do is trivial for $i=1$, so the reader may assume $i \geq 2$.

Our strategy is to study a quotient algebra of $\mathscr{E}$ which is Azumaya of constant rank $i^{2}$. All irreducible representations of such an algebra will be $i$-dimensional. Thus, if $U_{j}=\varnothing$ for $j<i$, but $U_{i} \neq \varnothing$, we will factor out the identities of $i \times i$ matrices, in order to get rid of all the irreducible representations in dimension greater than $i$.

Let $U=U_{i}$. Also note that $U_{j}=\varnothing$ for $j<i$ implies $U \subseteq \hat{M}_{i}^{n}(1.9)$; if $x \in U, x \in \check{M}_{i}^{n}$, then $x$ corresponds to a reducible $i$-dimensional representation, which yields a subrepresentation and a quotient representation in dimension less than $i$, contradicting the emptiness of $U_{j}, j<i$. We have the "Gelfand transformation": for $f \in \mathscr{E}, x \in U$, let $\hat{f}(x)=$ $\varphi(f)$, where $\varphi$ is the unique representation $\mathscr{E} \rightarrow M_{i}, X_{1} \mapsto x_{1}, \ldots, X_{n} \mapsto$ $x_{n}$.

4.8. Proposition. The map $f \mapsto \hat{f}$ defines a continuous homomorphism $\gamma: \mathscr{E} \rightarrow \mathscr{H}(U)=\mathscr{H}$.

Proof. [15, p. 15].

$\gamma$ is certainly not injective; $\operatorname{ker} \gamma$ contains $J$, the closure in $\mathscr{E}$ of the polynomial identities of $i \times i$ matrices.

\subsection{Definition. Let $\mathscr{G}=\mathscr{E} / J$.}

We obtain a homomorphism $\mathscr{G} \rightarrow \mathscr{H}$. We want to prove that $\mathscr{G} \rightarrow \mathscr{H}$ is in fact a (topological, as both $\mathscr{G}$ and $\mathscr{H}$ are Fréchet) isomorphism. 
We shall prove $(4.12,4.13)$ that $\mathscr{G}$ and $\mathscr{H}$ are Azumaya of rank $i^{2}$. Of course $\mathscr{G} \leq M_{i}$ (we factored out $J$ which contains the identities of $i \times i$ matrices) and $\mathscr{H} \leq M_{i}$ ( $\mathscr{H}$ is an algebra of $M_{i}$-valued functions). All closed maximal ideals of $\mathscr{G}$ and $\mathscr{H}$ correspond to points in $U \subseteq \hat{M}_{i}^{n}$, and they have codimension $i^{2}$. but we cannot apply Artin-Procesi (1.2) here, since $\mathscr{G}$ and $\mathscr{H}$ have maximal ideals that are not closed. We need the

4.10. Proposition. Let $A$ be a Fréchet algebra which is Azumaya over its center $Z$. Then the trace map [5, III §2] $T: A \rightarrow Z$ is continuous.

Proof. $A$ is a finite $Z$-module. Take $e_{1}, \ldots, e_{k}$ a generating set for $A$ over $Z$; if $a \in A, a=\sum_{j=1}^{k} a_{j} e_{j}$ for some $a_{1}, \ldots, a_{k} \in Z$. Since $T$ is $Z$-linear, $T(a)=\sum a_{j} T\left(e_{j}\right)$. Consider the map $\psi: Z^{k} \rightarrow A,\left(a_{1}, \ldots, a_{k}\right) \mapsto$ $\sum a_{j} e_{j} \cdot \psi$ is continuous and surjective, hence open, and $Z^{k} / \operatorname{ker} \psi \simeq A$ (as Fréchet spaces). Let $T^{\prime}: Z^{k} \rightarrow Z,\left(a_{1}, \ldots, a_{k}\right) \mapsto T\left(\sum a_{j} e_{j}\right)=\sum a_{j} T\left(e_{j}\right)$. $T^{\prime}$ is continuous and vanishes on $\operatorname{ker} \psi$, yielding a continuous map $T^{\prime \prime}$ : $Z^{k} / \operatorname{ker} \psi \rightarrow Z$. Now $T$ is just $T^{\prime \prime}$ provided we identify $A$ with $Z^{k} / \operatorname{ker} \psi$. (I am indebted to R. Brooks for this argument.)

Recall that we defined a map $\Pi: M_{i}^{n} \rightarrow \Delta \subseteq \mathbf{C}^{p}$ which sends $\hat{M}_{i}^{n}$ onto $\hat{\Delta}, \hat{\Delta}$ smooth (1.10). Since $U \subseteq \hat{M}_{l}^{n}$, we have $V=\Pi U \subseteq \hat{\Delta}, V$ is a smooth locally closed submanifold of $\mathbf{C}^{p}$, and

$$
\begin{aligned}
Z(\mathscr{H})=\left\{f: U \stackrel{\text { holom. }}{\rightarrow} \mathbf{C}, f\left(m x m^{-1}\right)\right. & =f(x) \text { whenever } \\
& \left.x \in U, m \in \mathrm{GL}_{i}\right\}=\mathcal{O}(V) .
\end{aligned}
$$

4.11. Proposition. $V$ is polynomially convex in $\mathbf{C}^{p}$.

Proof. Write $\mathscr{F}=\lim _{\leftarrow} \mathscr{F}_{k}$, where the $\mathscr{F}_{k}$ are Banach algebras, and $\mathscr{G}=\lim _{\leftarrow} \mathscr{G}_{k}$. Then each $\mathscr{G}_{k}$ is a Banach Azumaya algebra, as shown by (1.2): all maximal ideals of $\mathscr{G}_{k}$ are closed and have codimension $i^{2}$. Now $R$ (the algebra of $n$ generic $i \times i$ matrices) is dense in $\mathscr{G}$, for $F_{n+q}$ was dense in $\mathscr{F}$ (4.1) and density is preserved under quotients, and $R$ is dense in each $\mathscr{G}_{k}$. The trace map $T: \mathscr{G}_{k} \rightarrow Z\left(\mathscr{G}_{k}\right)$ is just (up to a coefficient which we may assume is 1) the usual trace, so $T$ maps $R$ into $B$, $B \subseteq Z\left(\mathscr{G}_{k}\right)$; since $R \subseteq \mathscr{G}_{k}$, we see that $B R=S \subseteq \mathscr{G}_{k}$. As $R$ is dense in $\mathscr{G}_{k}$, so is $S$. Furthermore, $B$ is dense in $Z\left(\mathscr{G}_{k}\right)$ : if $f \in Z\left(\mathscr{G}_{k}\right), f=T f, S$ dense in $\mathscr{G}_{k} \Rightarrow f=\lim f_{j} \quad\left(f_{j} \in S\right), \quad T$ continuous $(4.10) \Rightarrow f=T f=$ $\lim T f_{j}$, and each $T f_{j} \in Z(S)=B$. Since $B$ is dense in $Z\left(\mathscr{G}_{k}\right), Z\left(\mathscr{G}_{k}\right)$ is a 
finitely generated commutative Banach algebra. Its maximal ideal space $V_{k}$ can be identified with a compact, polynomially convex subset of $\hat{\Delta}$. Now $V_{k} \subseteq V$, in fact $V=\bigcup_{k} V_{k}$ is an increasing union of compact polynomially convex subsets of $\hat{\Delta}$, and each compact subset of $V$ is contained in some $V_{k}$. Therefore $V$ is polynomially convex.

We can now prove

\subsection{Proposition. $\mathscr{H}$ is an Azumaya algebra.}

Proof. In view of (1.3), it is sufficient to prove that $F(\mathscr{H})=Z(\mathscr{H})$. We already know that $Z(\mathscr{H})=\mathcal{O}(V)$. Let $\mathscr{C}$ be the set of central polynomials without constant term. Then $\check{M}_{i}^{n}=\bigcap\left\{f^{-1}(0)\right\}(f \in \mathscr{C})$. As $Z(R) \subset B$ and $B$ is Noetherian, we can suppose $\check{M}_{i}^{n}=\left\{x \in M_{i}^{n}: f_{1}(x)\right.$ $\left.=\cdots=f_{r}(x)=0\right\}$, with $f_{1}, \ldots, f_{r} \in B$. These functions $f_{1}, \ldots, f_{r}$ can be seen as functions on $\Pi M_{i}^{n}=\Delta$, they have no common zero on $\hat{\Delta}$, hence no common zero on $V$. As $V$ is polynomially convex, we can find $g_{1}, \ldots, g_{r} \in \mathcal{O}(V)$, with $f_{1} g_{1}+\cdots+f_{r} g_{r}=1$. This shows $1 \in F(\mathscr{H})$, and $\mathscr{H}$ is Azumaya.

Similarly, we have

\subsection{Proposition. $\mathscr{G}$ is an Azumaya algebra.}

Proof. The space of closed maximal ideals of $Z(\mathscr{G})$ is $V$ (same proof as in (4.11)). Take $f_{1}, \ldots, f_{r}$ as in (4.12). They have no common zero in $V$, and by a result of Arens [2] we can find $g_{1}, \ldots, g_{r} \in Z(\mathscr{G})$ with $f_{1} g_{1}$ $+\cdots+f_{r} g_{r}=1$. Thus $1 \in F(\mathscr{G})$ and $\mathscr{G}$ is Azumaya.

4.14. Remark. We have a map $\mathscr{G} \rightarrow \mathscr{H}$ between two Azumaya algebras of rank $i^{2}$. In order to show that this map is an isomorphism, it is enough to establish the isomorphism on the centers. Injectivity of $Z(\mathscr{G})$ $\rightarrow Z(\mathscr{H})$ implies injectivity of $\mathscr{G} \rightarrow \mathscr{H}$, because we have a natural correspondence between ideals of $\mathscr{G}$ and ideals of $Z(\mathscr{G})$. If $Z(\mathscr{G}) \rightarrow Z(\mathscr{H})$ is also surjective, then $\mathscr{G} \rightarrow \mathscr{H}$ is bijective [12, 1.8.49].

\subsection{Proposition. The map $Z(\mathscr{G}) \rightarrow Z(\mathscr{H})$ is an isomorphism.}

Proof. Again, write $\mathscr{G}=\lim _{\leftarrow} \mathscr{G}_{k}$ and $Z(\mathscr{G})=\lim _{\leftarrow} Z\left(\mathscr{G}_{k}\right)$. The maximal ideal space $V_{k}$ of $Z\left(\mathscr{G}_{k}\right)$ is a compact polynomially convex subset of $V$. By a form of the (commutative) holomorphic functional calculus due to Allan [1], there is a continuous homomorphism $\mathcal{O}(V) \rightarrow Z\left(\mathscr{G}_{k}\right)$ which is 
the identity on $B$. Passing to the projective limit, we obtain a map $\mathcal{O}(V) \rightarrow Z(\mathscr{G})$. We also have a map (Gelfand) $Z(\mathscr{G}) \rightarrow Z(\mathscr{H})=\mathscr{O}(V)$, and the composition $Z(\mathscr{G}) \rightarrow Z(\mathscr{H}) \rightarrow Z(\mathscr{G})$ is the identity on $B$, which is dense in $Z(\mathscr{G})$. Hence $Z(\mathscr{G}) \simeq Z(\mathscr{H})$.

From this, we obtain, in view of (4.14)

4.16. THEOREM. The map $\mathscr{G} \rightarrow \mathscr{H}$ is an isomorphism.

This theorem has several consequences

4.17. Corollary. $\mathscr{R}(U)=\mathscr{H}(U)$ (recall $(3.1,3.2)$ ) (for $R$ is dense in $\mathscr{H}(U)=\mathscr{G})$.

4.18. Corollary. The ideal $J$ (see 4.9) is equal to $\operatorname{ker} \gamma($ for $\mathscr{H}(U)$ is separated by its i-dimensional representations).

4.19. Proposition. There is a holomorphic bundle $\mathscr{M}$ over $V$ with fiber $M_{i}$ such that $\mathscr{H}$ is isomorphic with $\Gamma(V, \mathscr{M})$ the algebra of holomorphic sections of $\mathscr{M}$ over $V$.

Proof. Cover $U$ by open sets $U_{\alpha}$ such that $U_{\alpha} \simeq \Pi\left(U_{\alpha}\right) \times \mathrm{PGL}_{i}(\simeq$ as complex manifolds). Let $V_{\alpha}=\Pi U_{\alpha}$. On $U_{\alpha}$, $\Pi$ has a holomorphic section $\sigma_{\alpha}: V_{\alpha} \rightarrow U_{\alpha}, \Pi\left(\sigma_{\alpha}(z)\right)=z$ for all $z$ in $V_{\alpha}$. For $f \in \mathscr{H}(U)$, define $f_{\alpha}: V_{\alpha} \rightarrow M_{i}$ by $f_{\alpha}(z)=f\left(\sigma_{\alpha}(z)\right) \quad\left(z \in V_{\alpha}\right)$. We get homomorphisms $\mathscr{H}(U) \rightarrow \mathcal{O}\left(V_{\alpha}, M_{i}\right), f \mapsto f_{\alpha}$. Pasting these, we obtain a bundle $\mathscr{M}$ over $V$ and a map $\mathscr{H}(U) \rightarrow \Gamma(V, \mathscr{M})$. This map induces an isomorphism on the centers, and both algebras are Azumaya. Thus $\mathscr{H}(U) \simeq \Gamma(V, \mathscr{M})$.

\section{Chapter Five. Two Generic $2 \times 2$ Matrices}

Let $R$ be the algebra of generic $2 \times 2$ matrices generated by $X_{1}$ and $X_{2}$. The structure of $R$ is described in [7]. As generators for the algebra of invariants $B$, we take $z_{1}=\operatorname{tr} X_{1}, z_{2}=\operatorname{det} X_{1}, z_{3}=\operatorname{tr} X_{2}, z_{4}=\operatorname{det} X_{2}$, $z_{5}=\operatorname{tr} X_{1} X_{2}$, so $B \simeq \mathrm{C}\left[z_{1}, z_{2}, z_{3}, z_{4}, z_{5}\right]$. Note that

$$
\operatorname{det} X_{1}=\frac{1}{2}\left(\left(\operatorname{tr} X_{1}\right)^{2}-\operatorname{tr} X_{1}^{2}\right), \ldots,
$$

so all invariants could be expressed in terms of traces (1.8).

The algebra of concomitants $S$ is a free $B$-module of dimension 4: $S=B+B X_{1}+B X_{2}+B X_{1} X_{2}$. We shall also need the commutator ideal of $R$ (cf. 1.5)), [R,R] = [S,S] $S\left(X_{1} X_{2}-X_{2} X_{1}\right)$. 
We let $\tau=X_{1} X_{2}-X_{2} X_{1}, \sigma=\tau^{2}=\left(X_{1} X_{2}-X_{2} X_{1}\right)^{2}$, and $Z(R)=$ $\mathrm{C}+B \boldsymbol{\sigma}($ see 1.6).

We now find the lmc completion of $R$. As $\mathscr{R}=\mathscr{R}\left(M_{2}^{2}\right)$ is lmc and complete, we have an injective map $\tilde{R} \rightarrow \mathscr{R}$, and we shall show that it is a (topological, as both $\tilde{R}$ and $\mathscr{R}$ are Fréchet) isomorphism. Note that $\tilde{R}$ and $\mathscr{R}$ are not Azumaya (they have irreducible representations in dimension 1 and 2), so the method of Chapter Four (reduction to the center) will not do. Instead we are going to use a Five lemma argument.

We have a diagram with exact rows

$$
\begin{array}{lllllll}
0 \rightarrow \quad[\tilde{R}, \tilde{R}] & \rightarrow & \tilde{R} & \rightarrow & \tilde{R} /[\tilde{R}, \tilde{R}] \rightarrow 0 \\
& \alpha \downarrow & & \beta \downarrow & & \gamma \downarrow \\
0 \rightarrow \quad[\mathscr{R}, \mathscr{R}] & \rightarrow & \mathscr{R} & \rightarrow & \mathscr{R} /[\mathscr{R}, \mathscr{R}] \rightarrow 0
\end{array}
$$

where $[\tilde{R}, \tilde{R}]$ and $[\mathscr{R}, \mathscr{R}]$ are the closed commutator ideals of $\tilde{R}$ and $\mathscr{R}$. If we can prove that both $\alpha$ and $\gamma$ are isomorphisms, it will follow that $\beta$ is bijective too.

\subsection{Proposition. $\gamma$ is an isomorphism.}

Proof. Both $R /[\tilde{R}, \tilde{R}]$ and $\mathscr{R} /[\mathscr{R}, \mathscr{R}]$ are commutative Fréchet algebras generated by (the classes of) $X_{1}$ and $X_{2}$, and we have

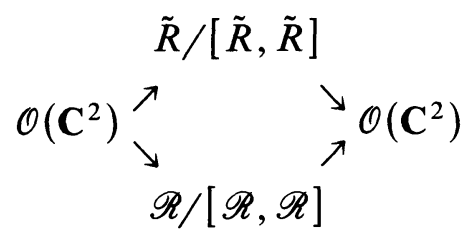

where the maps on the left are holomorphic functional calculi, and the maps on the right are Gelfand transforms. It follows that $\mathcal{O}\left(\mathbf{C}^{2}\right) \simeq$ $\tilde{R} /[\tilde{R}, \tilde{R}] \simeq \mathscr{R} /[\mathscr{R}, \mathscr{R}]$.

Now we have to show that $\alpha$ is an isomorphism. We let $\mathscr{B}=\tilde{B} \simeq$ $\mathscr{O}\left(\mathbf{C}^{5}\right)$, and define $\mathscr{S}=\mathscr{B}+\mathscr{B} X_{1}+\mathscr{B} X_{2}+\mathscr{B} X_{1} X_{2}$.

\subsection{Proposition. $\mathscr{S}$ is a free $\mathscr{B}$-module.}

Proof. If $a+b X_{1}+c X_{2}+d X_{1} X_{2}=0$, with $a, b, c, d \in \mathscr{B}$, write four scalar equatins (corresponding to the four matrix entries) and observe that the determinant of the system is $\sigma=\left(X_{1} X_{2}-X_{2} X_{1}\right)^{2}$. Since $\sigma$ is not a 
zero-divisor, $a=b=c=d=0$ is the only solution.

Next let

$$
\begin{aligned}
\mathscr{T}=\left\{f: M_{2}^{2} \stackrel{\text { hol. }}{\rightarrow} M_{2}, f\left(m x m^{-1}\right)=\right. & m f(x) m^{-1} \\
& \text { for all } \left.x \in M_{2}^{2}, m \in \mathrm{GL}_{2}\right\} .
\end{aligned}
$$

\subsection{Proposition. $\mathscr{S}=\mathscr{T}$.}

Proof. Clearly $\mathscr{S} \subseteq \mathscr{T}$, and $Z(\mathscr{S})=Z(\mathscr{T})=\mathscr{B}$. Let $f \in \mathscr{T}$. An argument similar to (3.3) allows us to write $f$ as the sum of a power series, all partial sums of which belong to $S=B+B X_{1}+B X_{2}+B X_{1} X_{2}$. Thus we can write $f=a+b X_{1}+c X_{2}+d X_{1} X_{2}$, where $a, b, c, d$ are formal power series in $z_{1}, z_{2}, z_{3}, z_{4}, z_{5}$. On the other hand, $a, b, c, d$ can also be computed by solving a $4 \times 4$ system with entries in $\mathscr{B}$ and determinant $\sigma$, so by Cramer's rule $a, b, c, d$ will be meromorphic functions: $a=A / \sigma$, $(A \in \mathscr{B})$, etc. But the fact that $a$ has a formal power series forces $a$ to be holomorphic on $\mathbf{C}^{5}$, i.e. $a \in \mathscr{B}$. Similarly, $b, c, d \in \mathscr{B}$ and $f \in \mathscr{S}$.

We let $\mathscr{K}=\mathscr{S} \tau$, and claim that $\mathscr{K}=[\tilde{R}, \tilde{R}]=[\mathscr{R}, \mathscr{R}]$. Actually, since $[\tilde{R}, \tilde{R}] \subseteq[\mathscr{R}, \mathscr{R}]$ we need only show that $\mathscr{K} \subseteq[\tilde{R}, \tilde{R}]$ and $[\mathscr{R}, \mathscr{R}] \subseteq \mathscr{K}$.

\subsection{Proposition. $\mathscr{K} \subseteq[\tilde{R}, \tilde{R}]$.}

Proof. It is enough to show that $\mathscr{B} \tau \subseteq[\tilde{R}, \tilde{R}]$.

Let $\nu$ be any submultiplicative seminorm on $R$. Without loss of generality, we can suppose $\nu$ is a norm. Let $E$ be the completion of $B \tau$ with respect to $\nu$. $E$ is a Banach space, and $\mathscr{L}(E)$ is a Banach algebra. We have an embedding $\psi: B \rightarrow \mathscr{L}(E)$, given by $(f \in B)$ :

$$
\begin{aligned}
& \psi\left(z_{1}\right)(f \tau)=X_{1} f \tau+f \tau X_{1} \\
& \psi\left(z_{2}\right)(f \tau)=X_{1} f \tau X_{1} \\
& \psi\left(z_{3}\right)(f \tau)=X_{2} f \tau+f \tau X_{2} \\
& \psi\left(z_{4}\right)(f \tau)=X_{2} f \tau X_{2} \\
& \psi\left(z_{5}\right)(f \tau)=X_{1} X_{2} f \tau+f \tau X_{1} X_{2} \quad \text { (see [7]). }
\end{aligned}
$$

These relations show that $\psi\left(z_{1}\right), \psi\left(z_{2}\right), \psi\left(z_{3}\right), \psi\left(z_{4}\right), \psi\left(z_{5}\right)$ are $\nu$ continuous $(B \tau \rightarrow B \tau)$, hence extend to continuous linear maps $E \rightarrow E$. Let $A$ be the closure of $\psi(B)$ in $\mathscr{L}(E) . A$ is a finitely generated 
commutative Banach algebra. At this point, we can employ the (commutative) holomorphic functional calculus: there is a continuous homomorphism $\mathcal{O}\left(\mathbf{C}^{5}\right)=\mathscr{B} \rightarrow A, 1 \mapsto 1, z_{1} \mapsto \psi\left(z_{1}\right), z_{2} \mapsto \psi\left(z_{2}\right), z_{3} \mapsto \psi\left(z_{3}\right), z_{4}$ $\mapsto \psi\left(z_{4}\right), z_{5} \mapsto \psi\left(z_{5}\right)$. Hence, if $f \in \mathscr{B}$, the linear map $\psi(f)$ will be in $A$, it will send $\tau$ onto $f \tau \in E$. Thus $\mathscr{B} \tau \subseteq E$. This holds for any choice of $\nu$, and $\mathscr{B} \tau \subseteq \overline{B \tau}$, the closure of $B \tau$ in $[\tilde{R}, \tilde{R}]$ (i.e., in $\tilde{R}$ ). We can multiply by $X_{1}, X_{2}$ and $X_{1} X_{2}$, proving that $\mathscr{K} \subseteq[\tilde{R}, \tilde{R}]$.

\subsection{Proposition. $[\mathscr{R}, \mathscr{R}] \subseteq \mathscr{K}$.}

Proof. $[\mathscr{R}, \mathscr{R}]=[\mathscr{H}, \mathscr{H}] \subseteq[\mathscr{T}, \mathscr{T}]=[\mathscr{S}, \mathscr{S}]=\mathscr{K}$

Combining (5.4) and (5.5), we have

5.6. Proposition. $[\tilde{R}, \tilde{R}]=[\mathscr{R}, \mathscr{R}]$, so $\alpha$ is an isomorphism.

Now, by the Five lemma, (5.1) and (5.6) yield

5.7. THEOREM. $\beta$ is an isomorphism, and $\tilde{R}=\mathscr{R}\left(M_{2}^{2}\right)=\mathscr{H}\left(M_{2}^{2}\right)$.

5.8. REMARK. We have not been able to find the lmc completion of the algebra of $n$ generic $i \times i$ matrices for $n>2$ or $i>2$. We believe that it should equal $\mathscr{R}\left(M_{i}^{n}\right)$, i.e., that $\mathcal{O}\left(M_{i}^{n}, M_{i}\right)$ induces the finest lmc topology on the algebra of $n$ generic $i \times i$ matrices. But it is only for $n=i=2$ that we have enough information on the generic matrix algebra (given in [7]) to prove it. The structure of the other generic matrix algebras is less well understood.

As an application of the above result, let us consider the following situation. $A$ is a topological algebra and $Z(A)$ its center. We have maps

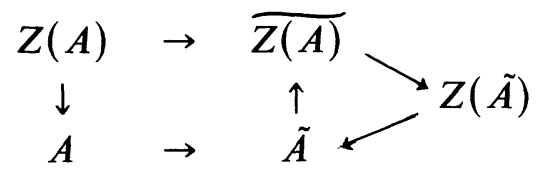

and the homomorphism $\overline{Z(A)} \rightarrow Z(\tilde{A})$ need not be one-to-one or onto, as shown by $(5.9,5.11)$.

5.9. Proposition. If $A$ is the algebra of two generic $2 \times 2$ matrices, the map $\overline{Z(A)} \rightarrow Z(\tilde{A})$ is not surjective.

Proof. $\tilde{A}=\mathscr{H}\left(M_{2}^{2}\right)=\mathscr{R}\left(M_{2}^{2}\right) \quad(5.7)$, and (cf. 1.6) $Z(\tilde{A})=\mathrm{C}+$ $\mathcal{O}\left(\mathbf{C}^{5}\right) \sigma$. Recall that $Z(A)=\mathbf{C}+B \sigma$. Endow $\mathbf{C}^{5}$ with a norm, and let 
$T=\left\{z \in \mathbf{C}^{5}:|\boldsymbol{\sigma}(z)| \leq \exp (-\|z\|)\right\} . T$ is ia "rapidly decreasing" neighborhood of $\left\{z \in \mathbf{C}^{5}: \sigma(z)=0\right\}$. $T$ is not compact. Nevertheless, every $f \in Z(A)$ is bounded on $T: f \in Z(A) \Rightarrow f=a+b \sigma(a \in \mathbf{C}, b \in B=$ $\left.\mathbf{C}\left[z_{1}, z_{2}, z_{3}, z_{4}, z_{5}\right]\right) ;|f(z)| \leq|a|+|b(z)| \exp (-\|z\|)$ for $z \in T$. We can define a submultiplicative seminorm on $Z(A)$ by $\nu(f)=\sup _{T}|f(z)| ; \nu$ does not extend to $Z(\tilde{A})$, as $g(z)=\sigma(z) \exp \left(z_{1}^{2}\right)$ is unbounded on $T$. So $\overline{Z(A)}$ is a proper subalgebra of $Z(\tilde{A})$.

5.10. REMARK. If it were true that $\overline{Z(A)}=\mathbf{C}+\mathcal{O}\left(\mathbf{C}^{5}\right) \sigma$, then it would be possible to show (using localization, in the ring theoretic sense) that $A=\mathscr{H}\left(M_{2}^{2}\right)$. This is the case, but for different reasons.

Here is a different phenomenon.

5.11. Proposition. If $A$ is the enveloping algebra of $\mathrm{sl}(2, \mathrm{C})$, then the map $\overline{Z(A)} \rightarrow Z(\tilde{A})$ is not injective.

Proof. By [14, p. 250], $\tilde{A}$ is a direct product of matrix algebras. We know that $A$ has one (up to equivalence) irreducible representation in each dimension 1 , so $\tilde{A} \simeq \prod_{i=1}^{\infty} M_{i}$, and $Z(\tilde{A}) \simeq \mathbf{C}^{\mathbf{N}}$ is the algebra of sequences of complex numbers. Now $Z(A)=\mathrm{C}[\Omega]$ ( $\Omega$ is "the" Casimir element), and $\overline{Z(A)} \simeq \mathcal{O}(\mathbf{C})$, the entire functions of $\Omega$. We have the map $\varphi: \overline{Z(A)} \rightarrow Z(\tilde{A})$. Looking at the infinitesimal character, we see that $\varphi$ sends the function $\Omega \in \mathcal{O}(\mathbf{C})$ to the sequence $(0,3 / 4,2, \ldots$, $\left.\left(n^{2}-1\right) / 4, \ldots\right) \in \mathbf{C}^{\mathbf{N}}$. Hence

$$
\varphi(f)=\left(f(0), f(3 / 4), \ldots, f\left(\left(n^{2}-1\right) / 4\right), \ldots\right) .
$$

By the Weierstrass theorem [8, 1.5.4], $\varphi$ is a surjection $\mathcal{O}(\mathbf{C}) \rightarrow \mathbf{C}^{\mathbf{N}} . \varphi$ is not injective, for its kernel contains $f(\Omega)=\sin 4 \pi \Omega$.

\section{Chapter Six. The Functional Calculus}

The results of Chapter Four will now yield a functional calculus for topological subquotients of Banach Azumaya algebras, giving a positive answer to Taylor's question: if $A$ is a separated quotient of a closed (unital) subalgebra of a Banach Azumaya algebra, if $a=\left(a_{1}, \ldots, a_{n}\right) \in A^{n}$, then there is a continuous algebra homomorphism $\mathscr{D}(\Omega) \rightarrow A$ that sends $1 \mapsto 1, X_{1} \mapsto a_{1}, \ldots, X_{n} \mapsto a_{n}$. If we suppose that $A$ is a closed subalgebra of a Banach Azumaya algebra, we can even get a functional calculus on a polynomial polyhedron. We will also give a result valid for two variables in any Banach algebra satisfying all identities of $2 \times 2$ matrices. 
First let $A$ be a Banach Azumaya algebra of rank $i^{2}$ over its center $Z(A)$. Take $\left(a_{1}, \ldots, a_{n}\right)=a \in A^{n}$. Our idea is the following: choosing suitable free polynomials $p_{1}, \ldots, p_{q}$, radii $r_{1}, \ldots, r_{n}, s_{1}, \ldots, s_{q}$, using the implicit function theorem of Chapter Four, we will get a homomorphism $\mathscr{G} \rightarrow \mathscr{H}(U)$ which, provided $\mathscr{G}$ and $\mathscr{H}$ are Azumaya, will be an isomorphism (4.16). Since $\mathscr{G}$ maps into $A$, we will obtain a continuous homomorphism $\mathscr{H} \rightarrow A$. But things are not that simple. Since all irreducible representations of $A$ are $i$-dimensional, we have have (see 1.13) that $\operatorname{sp}_{j}(a)=\varnothing$ for $j<i$. This does not imply that $\operatorname{sp}_{i}(a) \subseteq \hat{M}_{i}^{n}$ (a trivial counterexample: let $a_{1}=\cdots=a_{n}=0$ ). Even if $\operatorname{sp}_{i}(a) \subseteq \hat{M}_{i}^{n}$, we do not know that $p \operatorname{sp}_{i}(a) \subseteq \hat{M}_{i}^{n}$ (see 1.15), and even if $p \operatorname{sp}_{i}(a) \subseteq \hat{M}_{i}^{n}$, can we find suitable $p_{1}, \ldots, p_{q}$ and corresponding radii such that $U_{i}=U \subseteq \hat{M}_{i}^{n}$ ? If this can be done, then the machinery of Chapter Four will apply. The difficulties can be overcome. As $A$ is Azumaya, $Z(A)=F(A)(1.3)$; in particular, $1 \in F(A)$, there exists $P_{0}$, a free polynomial without constant term which is central for $i \times i$ matrices, and $b_{1}, \ldots, b_{m} \in A$, such that $P_{0}\left(b_{1}, \ldots, b_{m}\right)=P_{0}(b)=1$. Let $p_{0}=1-P_{0}$. Let us use the construction of Chapter Four, $p_{1}, \ldots, p_{q}$ being free polynomials in $X_{1}, \ldots, X_{n}, p_{0}$ a free polynomial in $X_{1}^{\prime}, \ldots, X_{m}^{\prime}$. We choose $r_{1}>\left\|a_{1}\right\|, \ldots, r_{n}>\left\|a_{n}\right\|, s_{1}>$ $\left\|p_{1}(a)\right\|, \ldots, s_{q}>\left\|p_{q}(a)\right\|$. We take $\mathscr{F}^{+}$to be the free power series algebra in $X_{1}, \ldots, X_{n}, X_{1}^{\prime}, \ldots, X_{n}^{\prime}, Y_{1}, \ldots, Y_{q}, Y_{0}$, with the radii $r_{1}, \ldots, r_{n}$, $\infty, \ldots, \infty, s_{1}, \ldots, s_{q}, 1$. We have a continuous homomorphism (2.4) $\mathscr{F}^{+} \rightarrow A, \quad 1 \mapsto 1, \quad X_{1} \mapsto a_{1}, \ldots, X_{n} \mapsto a_{n}, \quad X_{1}^{\prime} \mapsto b_{1}, \ldots, X_{m}^{\prime} \mapsto b_{m}, \quad Y_{1} \mapsto$ $p_{1}(a), \ldots, Y_{q} \mapsto p_{q}(a), \quad Y_{0} \mapsto 0=1-P_{0}(b)=p_{0}(b)$. This homomorphism vanishes on the closed ideal generated in $\mathscr{F}^{+}$by $Y_{0}-p_{0}, Y_{1}-$ $p_{1}, \ldots, Y_{q}-p_{q}$, and by all polynomial identities of ' Letting $\mathscr{G}^{+}$be the quotient, we obtain a map $\mathscr{G}^{+} \rightarrow A, \quad X_{1} \mapsto a_{1}, \ldots, X_{n} \mapsto a_{n}, \quad X_{1}^{\prime} \mapsto$ $b_{1}, \ldots, X_{m}^{\prime} \mapsto b_{m}$. Let

$$
U^{+}=\left\{\left(x_{1}, \ldots, x_{n}, x_{1}^{\prime}, \ldots, x_{m}^{\prime}\right) \in M_{i}^{n+m}:\right. \text { there is a representation }
$$

$$
\left.\mathscr{G}^{+} \rightarrow A, X_{1} \mapsto x_{1}, \ldots, X_{n} \mapsto x_{n}, X_{1}^{\prime} \mapsto x_{1}^{\prime}, \ldots, X_{m}^{\prime} \mapsto x_{m}^{\prime}\right\} .
$$

Now $U^{+}=U \times U^{\prime}$, where $U \subseteq M_{i}^{n}, U^{\prime} \subseteq M_{i}^{m}$; in fact (4.6) $U^{\prime}=$ $\left\{\left(x_{1}^{\prime}, \ldots, x_{m}^{\prime}\right) \in M_{i}^{m}:\left|p_{0}\left(x^{\prime}\right)\right|<1\right\}$, and $U^{\prime} \subseteq \hat{M}_{i}^{m}$ (for $P_{0}=1-p_{0}$ vanishes on $\left.\check{M}_{i}^{m}\right)$, hence $U \subseteq \hat{M}_{i}^{n+m}$. By (4.16), the map $\mathscr{G}^{+} \rightarrow \mathscr{H}^{+}=$ $\mathscr{H}\left(U^{+}\right)$is an isomorphism. This shows

6.1. Proposition. There is a unique continuous homomorphism $\mathscr{H}\left(U^{+}\right)$ $\rightarrow A, 1 \mapsto 1, X_{1} \mapsto a, \ldots, X_{n} \mapsto a_{n}, X_{1}^{\prime} \mapsto b_{1}, \ldots, X_{m}^{\prime} \mapsto b_{m}$. (Uniqueness follows from polynomial density (4.17)). 
This is not satisfactory yet, because

(a) we do not know $U^{+}$in terms of analytic inequalities;

(b) we are interested in functions of $a, \ldots, a_{n}$, and $b_{1}, \ldots, b_{m}$ have been thrown in.

In order to get rid of the $b_{1}, \ldots, b_{m}$, observe that $\mathscr{H}\left(U^{+}\right)=\mathscr{R}\left(U^{+}\right)$. Restrict the map $\mathscr{H}\left(U^{+}\right) \mapsto A$ to the algebra of $n$ generic matrices generated by $X_{1}, \ldots, X_{n}$, and extend it by continuity to the closure, viz. $\mathscr{R}(U)$ (recall that $U^{+}$is a product domain). This implies

6.2. Proposition. There is a (unique) continuous homomorphism $\mathscr{R}(U) \rightarrow A, 1 \mapsto 1, X_{1} \mapsto a_{1}, \ldots, X_{n} \mapsto a_{n}$.

All we know about $U$ is (4.5). We let

$$
\begin{aligned}
U^{*}=\left\{x \in M_{i}^{n}: \rho\left(x_{1}\right)<r_{1}, \ldots, \rho\left(x_{n}\right) \leq r_{n},\right. \\
\\
\left.\quad \rho p_{1}(x)<s_{1}, \ldots, \rho p_{q}(x)<s_{q}\right\}
\end{aligned}
$$

Then $U^{*} \supseteq U(4.4)$, which gives a homomorphism $\mathscr{R}\left(U^{*}\right) \rightarrow \mathscr{R}(U)$, and (6.2) yields

6.3. Proposition. There is a unique continuous homomorphism $\mathscr{R}\left(U^{*}\right)$ $\rightarrow A, 1 \mapsto 1, X_{1} \mapsto a_{1}, \ldots, X_{n} \mapsto a_{n}$.

The proposition is not quite optimal: we chose $r_{1}>\left\|a_{1}\right\|, \ldots, r_{n}>$ $\left\|a_{n}\right\|, s_{1}>\left\|p_{1}(a)\right\|, \ldots, s_{q}>\left\|p_{q}(a)\right\|$; and we define $U^{*}$ by spectral radii. Fortunately, we can express everything in terms of spectral radii. Take $\bar{r}_{1}>\rho\left(a_{1}\right), \ldots, \bar{r}_{n}>\rho\left(a_{n}\right), \bar{s}_{1}>\rho p_{1}(a), \ldots, \bar{s}_{q}>\rho p_{q}(a)$; then for $k$ large enough, $\left\|a_{1}^{k}\right\|<\bar{r}_{1}^{k}, \ldots,\left\|a_{n}^{k}\right\|<\bar{r}_{n}^{k},\left\|p_{1}^{k}(a)\right\|<\bar{s}_{1}^{k}, \ldots,\left\|p_{q}^{k}(a)\right\|<\bar{s}_{q}^{k}$. We just repeat the whole construction, but with $(n+q)$ extra polynomials $X_{1}^{k}, \ldots, X_{n}^{k}, p_{1}^{k}, \ldots, p_{q}^{k}$, and radii $\bar{r}_{1}^{k}, \ldots, \bar{r}_{n}^{k}, \bar{s}_{1}^{k}, \ldots, \bar{s}_{q}^{k}$. Since $\rho\left(x_{1}^{k}\right)=$ $\rho\left(x_{1}\right)^{k}, \ldots,(6.3)$ becomes

6.4. THEOREM. If

$$
\begin{aligned}
W=\left\{x \in M_{i}^{n}: \rho\left(x_{1}\right)\right. & <\bar{r}_{1}, \ldots, \rho\left(x_{n}\right)<\bar{r}_{n}, \\
& \left.\rho p_{1}(x)<\bar{s}_{1}, \ldots, \rho p_{q}(x)<\bar{s}_{q}\right\},
\end{aligned}
$$

there is a unique continuous homomorphism $\mathscr{R}(W) \rightarrow A, X_{1} \mapsto a_{1}, \ldots, X_{n}$ $\mapsto a_{n}$ 
This gives the functional calculus on polynomial polyhedra for Banach Azumaya algebras of constant rank. Now let $A_{0}$ be a closed unital subalgebra of the Banach algebra $A$, Azumaya of rank $i^{2}$. Let $a, \ldots, a_{n} \in$ $A_{0}$. We have

6.5. Proposition. With $W$ as in (6.4), there is a unique continuous homomorphism $\mathscr{R}(W) \rightarrow A_{0}, 1 \mapsto 1, X_{1} \mapsto a_{1}, \ldots, X_{n} \mapsto a_{n}$.

Proof. This follows immediately from (6.4), since $A_{0}$ is closed in $A$.

6.6. Proposition. If $A_{0}$ is a Banach Azumaya algebra, it is a closed subalgebra of a Banach Azumaya algebra of constant rank.

Proof. Let $A_{0}=\oplus_{j=1}^{i} A_{j}, A_{j}$ Azumaya of rank $j^{2}$. Let $k=i$ ! We can embed $A_{j}$ as a closed subalgebra of $M_{k / j}\left(A_{j}\right)$ which is Azumaya of rank $k^{2}$, and $A_{0} \simeq \bigoplus_{j=1}^{i} M_{k / j}\left(A_{j}\right)$; the embedding has closed range, for the sum is direct.

6.7. Corollary. Proposition 6.5 holds for closed subalgebras of Banach Azumaya algebras (of nonconstant rank).

Now let $A$ be a Banach Azumaya algebra, $A_{0}$ a closed subalgebra of $A$, and $A_{1}$ the quotient of $A_{0}$ by a closed ideal. Let $\left(a, \ldots, a_{n}\right) \in A_{1}^{n}$. Lift it to $\left(\bar{a}_{1}, \ldots, \bar{a}_{n}\right) \in A_{0}^{n}$.

6.8. Proposition. If $r_{1}>\rho\left(\bar{a}_{1}\right), \ldots, r_{n}>\rho\left(\bar{a}_{n}\right), s_{1}>\rho p_{1}(\bar{a}), \ldots, s_{q}$ $>\rho p_{q}(\bar{a})$, if

$$
\begin{aligned}
W=\left\{x \in M_{i}^{n}: \rho\left(x_{1}\right)\right. & <r_{1}, \ldots, \rho\left(x_{n}\right)<r_{n}, \\
& \left.\rho p_{1}(x)<s_{1}, \ldots, \rho p_{q}(x)<s_{q}\right\},
\end{aligned}
$$

there is a unique continuous homomorphism $\mathscr{R}(W) \rightarrow A_{1}, 1 \mapsto 1, X_{1} \mapsto$ $a_{1}, \ldots, X_{n} \mapsto a_{n}$.

Proof. Compose $\mathscr{R}(W) \rightarrow A_{0}(6.5)$ with the quotient map $A_{0} \rightarrow A_{1}$.

This proposition is not fully satisfactory: we had to take $r_{1}>$ $\rho\left(\bar{a}_{1}\right), \ldots$ We do not know if it holds with $r_{1}>\rho\left(a_{1}\right), \ldots$ Anyway, (6.8) gives us the

6.9. Corollary. If $A_{1}$ is a topological subquotient of a Banach Azumaya algebra of rank $i^{2}$, and $a, \ldots, a_{n} \in A_{1}$, there is a unique continuous homomorphism $\mathscr{R}\left(M_{i}^{n}\right) \rightarrow A_{1}, 1 \mapsto 1, X_{1} \mapsto a_{1}, \ldots, X_{n} \mapsto a_{n}$. 
We are ready for a partial answer to Taylor's question.

6.10. Proposition. If $A_{1}$ is a topological subquotient of a Banach Azumaya algebra, if $a, \ldots, a_{n} \in A_{1}$, there is a unique continuous homomorphism $\mathscr{D}(\Omega) \rightarrow A_{1}, 1 \mapsto 1, X_{1} \mapsto a_{1}, \ldots, X_{n} \rightarrow a_{n}$.

Proof. Compose $\mathscr{D}(\Omega) \rightarrow \mathscr{R}\left(M_{i}^{n}\right) \rightarrow A_{1}$. To see that $\mathscr{D}(\Omega) \rightarrow \mathscr{R}\left(M_{i}^{n}\right)$, combine (2.11) and the argument of (3.3).

6.11. REMARK. It is not known if every finitely generated Banach PI-algebra is a topological subquotient of a Banach Azumaya algebra. In a purely algebraic setting, every algebra with $n$ generators that satisfies some identity is a quotient of the algebra $R$ of $n$ generic $i \times i$ matrices for some $i$ (1.1), and $R$ can be presented as a subalgebra of a matrix (hence Azumaya) algebra. In the Banach case, the best we can say is

6.12. Proposition. Let $A \leq M_{i}$ be a unital Banach algebra. Assume $a \in F(A)$ and $a$ is not a topological divisor of zero. Then $A$ can be embedded as a closed subalgebra of a Banach algebra $B$ which is Azumaya of rank $i^{2}$.

Proof. Since $a$ is not a topological divisor of zero, there is a Banach algebra $B$ that isometrically contains $A$ and where $a$ is invertible [3] (here $A$ is not commutative, but $a$ is central, and Aren's proof works in our case). Now $a \in F(B), a$ invertible in $B$ imply $1 \in F(B)$, and $B$ is Azumaya.

We now employ the results of Chapter Five.

Let $A$ be a unital Banach algebra, $A \leq M_{2}$; take $\left(a_{1}, a_{2}\right) \in A^{2}$.

6.13. Proposition. There is a unique continuous homomorphism $\mathscr{R}\left(M_{2}^{2}\right) \rightarrow A, 1 \mapsto 1, X_{1} \mapsto a_{1}, X_{2} \mapsto a_{2}$.

Proof. This is just a restatement of (5.7).

6.14. CoROllaRY. There is a continuous homomorphism $\mathscr{D}(\Omega) \rightarrow A$, $1 \mapsto 1, X_{1} \mapsto a_{1}, X_{2} \mapsto a_{2}$

Let us summarize $(2.7,6.10,6.14)$. 
6.15. TheOREM. Let $A$ be a unital Banach algebra; $a, \ldots, a_{n} \in A$. In order for a continuous homomorphism $\mathscr{D}(\Omega) \rightarrow A, 1 \mapsto 1, X_{1} \rightarrow a_{1}, \ldots, X_{n}$ $\mapsto a_{n}$ to exist, it is necessary that $B$, the closed subalgebra of $A$ generated by $1, a, \ldots, a_{n}$ satisfy a polynomial identity; it is sufficient that $B$ be $a$ topological subquotient of a Banach Azumaya algebra or that $n=2$ and $B \leq M_{2}$. We conjecture that the necessary condition is also sufficient.

In Chapter One, we defined various notions of spectrum, including the "polynomial spectrum" (1.15). We can restate (6.5) in terms of $p \mathrm{sp}$. First we need a

6.16. Proposition. Let $A$ be a unital Banach PI-algebra, and $b \in A$. Then $\rho(b)=\sup \{\rho \varphi(b): \varphi$ irreducible representation of $A\}$.

Proof. It is obvious that $\rho(b) \geq \rho \varphi(b)$ for all $\varphi$. Now there is $s \in \mathbf{C}$, $|s|=\rho(b)$, such that $(b-s)$ is not invertible, hence not invertible on either side [9, II.4.3]. Thus $A(b-s)$ is a proper left ideal, which is contained in a maximal left ideal $I$. This gives an irreducible (finite-dimensional, 1.12) representation $\varphi$ of $A$. As $\varphi(b-s)=\varphi(b)-s$ is not invertible, $\rho \varphi(b) \geq|s|=\varphi(b)$.

Take $p_{1}, \ldots, p_{q}$ free polynomials, $r_{1}, \ldots, r_{n}, s_{1}, \ldots, s_{q}>0$. An open set of the form

$$
\begin{aligned}
W=\left\{x \in M_{i}^{n}: \rho\left(x_{1}\right)\right. & <r_{1}, \ldots, \rho\left(x_{n}\right)<r_{n}, \\
& \left.\rho p_{1}(x)<s_{1}, \ldots, \rho p_{q}(x)<s_{q}\right\}
\end{aligned}
$$

is called a polynomial polyhedron.

6.17. Proposition. Let $A$ be a Banach algebra that is Azumaya of rank $i^{2}$ over its center. Let $\left(a, \ldots, a_{n}\right)=a \in A^{n}$. If $W \subseteq M_{i}^{n}$ is a polynomial polyhedron that contains $\operatorname{sp}_{i}(a)=f \operatorname{sp}_{i}(a)$, there is a continuous homomorphism $\mathscr{R}(W) \rightarrow A, 1 \mapsto 1, X_{1} \mapsto a_{1}, \ldots, X_{n} \rightarrow a_{n}$.

Proof. Recall (6.4). All we have to show is that $W \supseteq \operatorname{sp}_{i}(a)$ implies $r_{1}>\rho\left(a_{1}\right), \ldots, r_{n}>\rho\left(a_{n}\right), s_{1}>\rho p_{1}(a), \ldots, s_{q}>\rho p_{q}(a)$. By (6.16), $\rho\left(a_{1}\right)$ $=\sup \left\{\rho \varphi\left(a_{1}\right): \varphi\right.$ irreducible $\}$. So

$$
\begin{aligned}
\operatorname{sp}_{i}(a) & \subseteq W \Rightarrow\left(\varphi\left(a_{1}\right), \ldots, \varphi\left(a_{n}\right)\right) \in W \quad(\text { all } \varphi) \\
& \Rightarrow \rho \varphi\left(a_{1}\right)<r_{1} \quad(\text { all } \varphi) \\
& \Rightarrow \rho\left(a_{1}\right)<r_{1}, \ldots
\end{aligned}
$$


Here is the corresponding formulation of (6.5). Let $A_{0}$ be a closed subalgebra of $A$, a Banach Azumaya of rank $i^{2}$. In view of (6.6), we can assume that the dimension of all irreducible representations of $A_{0}$ divides $i$ (this is automatic when $i=2$ ). Take $a=\left(a, \ldots, a_{n}\right) \in A_{0}^{n}$.

6.18. Proposition. If $W$ is a polynomial polyhedron that contains $f \operatorname{sp}_{i}(a)$, there is a continuous homomorphism $\mathscr{R}(W) \rightarrow A_{0}, 1 \mapsto 1, X_{1} \mapsto$ $a_{1}, \ldots, X_{n} \mapsto a_{n}$.

Proof. Similar to (6.17), as every irreducible representation yields a $i$-dimensional representation.

6.19. REMARK. In the commutative case, the Arens-Calderon trick [8, 3.2.3] allows us to obtain a functional calculus on (an open neighborhood of) the spectrum. For PI-algebras, it is not clear that we can pass from polynomial polyhedra to arbitrary open sets containing the full spectrum. Every commutative algebra with unit "contains" C, so the resolvent equation $\sum_{k=1}^{n}\left(a_{k}-s_{k}\right) x_{k}=1$ makes sense, and is solvable precisely for $s \notin \mathrm{sp}(a)$. For $A \leq M_{i}$, there is in general no embedding $M_{i} \rightarrow A$, and the problem should be formulated differently.

\section{REFERENCES}

[1] G. Allan, On lifting analytic relations in commutative Banach algebras, J. Funct. Anal., 5 (1970), 37-43.

[2] R. Arens, Dense inverse limit rings, Michigan Math. J., 5 (1958), 169-182.

[3] _ Inverse producing extensions of normed algebras, Trans. Amer. Math. Soc., 88 (1958), 536-548.

[4] A. Braun, The radical in a finitely generated PI-algebra, Bull. Amer. Math. Soc., (New Series) 7 (1982), 385-386.

[5] F. DeMeyer and E. Ingraham, Separable Algebras Over Commutative Rings, Springer Lecture Notes in Math., 181 (1971).

[6] J. Fell, The structure of algebras of operator fields, Acta Math., 106 (1961), 233-280.

[7] E. Formanek, P. Halpin and W. C. W. Li, The Poincare series of the ring of $2 \times 2$ generic matrices, J. Algebra, 69 (1981), 105-112.

[8] L. Hormander, An Introduction to Complex Analysis in Several Variables, North Holland, Amsterdam (1973).

[9] C. Procesi, Rings With Polynomial Identities, M. Dekker, New York (1973).

[10] __ Representations of algebras, Israel J. Math., 19 (1974), 169-182.

[11] _ The invariant theory of $n \times n$ matrices, Adv. Math., 19 (1976), 306-381.

[12] L. Rowen, Polynomial Identities in Ring Theory, Academic Press, New York (1980).

[13] J. Taylor, Homology and cohomology for topological algebras, Adv. Math., 9 (1972), 137-182. 
[14] _ , A general framework for a multi-operator functional calculus, Adv. Math., 9 (1972), 183-252.

[15] _ Functions of several noncommuting variables, Bull. Amer. Math. Soc., 79 (1973), 1-34.

[16] F. Van Oystaeyen and A. Verschoren, Non-commutative Algebraic Geometry, Springer Lecture Notes in Math., 887 (1981).

Received January 9, 1985.

UNIVERSITE LIBRE DE BRUXELLES

C.P. 214, BOULEVARD DU TRIOMPHE

1050 BRUXELLES, BELGIUM 


\section{PACIFIC JOURNAL OF MATHEMATICS EDITORS}

\author{
V. S. VARADARAJAN \\ (Managing Editor) \\ University of California \\ Los Angeles, CA 90024 \\ HERBERT ClEMENS \\ University of Utah \\ Salt Lake City, UT 84112 \\ R. FINN \\ Stanford University \\ Stanford, CA 94305
}

\author{
HERMANN FLASCHKA \\ University of Arizona \\ Tucson, AZ 85721 \\ RAMESH A. GANGOLLI \\ University of Washington \\ Seattle, WA 98195 \\ VAUghan F. R. JONES \\ University of California \\ Berkeley, CA 94720 \\ ROBION KIRBY \\ University of California \\ Berkeley, CA 94720
}

C. C. MOORE

University of California Berkeley, CA 94720

H. SAMELSON

Stanford University Stanford, CA 94305

HAROLD STARK

University of California, San Diego La Jolla, CA 92093

\section{ASSOCIATE EDITORS}
R. ARENS
E. F. BECKENBACH
B. H. NEUMANN
F. WOLF
K. YOSHIDA (1906-1982)

\section{SUPPORTING INSTITUTIONS}

UNIVERSITY OF ARIZONA

UNIVERSITY OF BRITISH COLUMBIA

CALIFORNIA INSTITUTE OF TECHNOLOGY

UNIVERSITY OF CALIFORNIA

MONTANA STATE UNIVERSITY

UNIVERSITY OF NEVADA, RENO

NEW MEXICO STATE UNIVERSITY

OREGON STATE UNIVERSITY
UNIVERSITY OF OREGON UNIVERSITY OF SOUTHERN CALIFORNIA

STANFORD UNIVERSITY

UNIVERSITY OF HAWAII

UNIVERSITY OF TOKYO

UNIVERSITY OF UTAH

WASHINGTON STATE UNIVERSITY

UNIVERSITY OF WASHINGTON 


\section{Pacific Journal of Mathematics}

Vol. 125, No. 1 September, 1986

Gilles Christol, Fonctions et éléments algébriques $\ldots \ldots \ldots \ldots \ldots \ldots \ldots \ldots$

Jo-Ann Deborah Cohen, Extensions of valuation and absolute valued

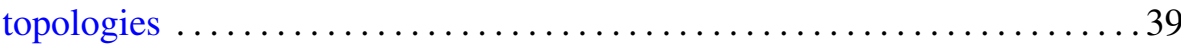

Miriam Cohen, Smash products, inner actions and quotient rings . . . . . . 45

Mikio Furushima, On the singular $K-3$ surfaces with hypersurface

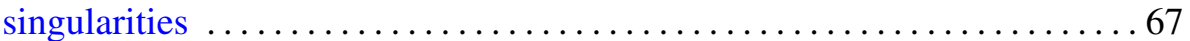

Gerhard Gierz and Boris Shekhtman, A duality principle for rational

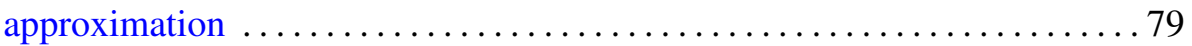

Anthony Wood Hager, A description of HSP-like classes, and

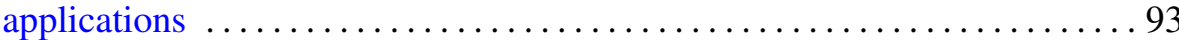

George Alan Jennings, Lines having high contact with a projective

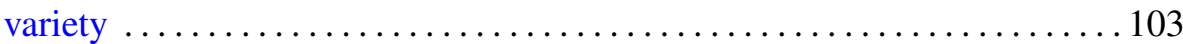

John Lott, Eigenvalue bounds for the Dirac operator . . . . . . . . . . . 117

Denis Laurent Luminet, A functional calculus for Banach PI-algebras . . . . 127

Shizuo Miyajima and Noboru Okazawa, Generators of positive

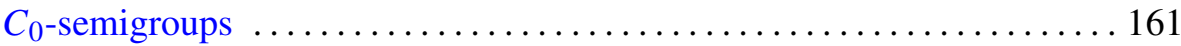

Takemi Mizokami, On functions and stratifiable $\mu$-spaces $\ldots \ldots \ldots \ldots \ldots 177$

Jeff Parker, 4-dimensional $G$-manifolds with 3-dimensional orbits . . . . . 187

Elias Saab and Paulette Saab, On Peł czyński's properties (V) and (V*) . . 205

Elmar Schrohe, The symbols of an algebra of pseudodifferential operators

Aart van Harten and Els Vader-Burger, Approximate Green functions as a tool to prove correctness of a formal approximation in a model of competing and diffusing species

Stephen Watson, Using prediction principles to construct ordered

continua 